\title{
New criteria on exponential synchronization and existence of periodic solutions of complex BAM networks with delays
}

\author{
Chao Yang ${ }^{\mathrm{a}}$, Lihong Huang ${ }^{\mathrm{b}, *}$ \\ ${ }^{a}$ Department of Mathematics and Computer Science, Changsha University, Changsha 410022, P. R. China. \\ ${ }^{b}$ School of Mathematical and Statistics, Changsha University of Science and Technology, Changsha, Hunan 410114, P. R. China. \\ Communicated by M. De La Sen
}

\begin{abstract}
In this paper, we study a class of time-delayed BAM neural networks with discontinuous activations. Base on the framework of differential inclusion theory and set-valued analysis, by designing discontinuous feedback controller and using some analytic methods, easily verifiable delay-independent criteria are established to guarantee the existence of periodic solution and global exponential synchronization of the drive-response system. Finally, we give a numerical example to illustrate our theoretical analysis. The obtained results are essentially new and they extend previously known results. (c)2017 All rights reserved.
\end{abstract}

Keywords: BAM neural networks, time-delayed, discontinuous activations, periodic solution, exponential synchronization. 2010 MSC: 34D06, 34C25, 34A36.

\section{Introduction}

As far as we know, many neural network systems such as Hopfield neural networks, Cohen-Grossberg neural networks and Bi-directional associative memory (BAM) neural networks were widely investigated in past decades, the BAM neural networks which generalized the single-layer auto-associative Hebbian correlator to a two-layer firstly introduced by Kosko (see $[15,16])$. Recently, BAM neural networks as well as their various promotions have attracted more attention of many researchers like mathematicians, biologist, physicists and computer scientists (see [4, 17, 20, 22, 23, 27, 29, 30]), because the BAM network structure can lead to better results in their potential applications in associative memory, nonlinear optimization problems and parallel computation than the regular neural network structures.

For given vector field, many researches of dynamical behaviors are established under the conditions of smoothness or even globally Lipschitz, because of that the solutions of continuous system are conventional definition and simple existence assumptions. In real world, however, it is known that neural networks with discontinuous activations do frequently arise. In order to ensure satisfaction degrees, in the conducting region, those input-output activation functions are asked to possess a very high slope, then they should approximate some discontinuous activation functions (see $[5,9,19,26])$. Recently, a

\footnotetext{
*Corresponding author

Email addresses: yang0915@hnu.edu.cn (Chao Yang), Ihhuang@csust.edu.cn (Lihong Huang)
}

doi:10.22436/jnsa.010.10.29 
newfangled tool named Filippov differential inclusion theory has been studied to investigate the dynamical behaviors of BAM system such as periodic solution, almost periodic solution, equilibrium point, convergence of solution and synchronization in the sense of Filippov. According to what we know, some experiments have been made to investigate the periodic synchronization problems for nonlinear systems with discontinuous dynamic behaviors in the field of neural networks, discontinuous controllers can be intentionally designed to realize synchronization stability. In [31], the authors investigated finite-time synchronization of an array of coupled neural networks via discontinuous controllers. In [6], the authors studied global exponential synchronization of the drive-response network system by designed novel discontinuous switching state-feedback control. It should be noted that it still has a lot of difficulties and lack of new and valid methods for realizing synchronization control of discontinuous neural networks in practice.

To the best of our knowledge, there are few papers published on the existence and exponential synchronization of periodic solution of complex BAM neural networks with time-varying delays and discontinuous activations. In fact, the classical controller without switching term is not very easy to realize the exponential stability of discontinuous models, because it can not be well handled the equivocal differences between the Filippov solutions of discontinuous neural network systems. Thus, it is necessary and important to study the existence and stability of periodic solution for neutral type neural networks with time-varying delays and discontinuous activations. In this paper, by designing a novel discontinuous controller including a discontinuous function sign(-), we will introduce the framework of differential inclusion theory and set-valued analysis to overcome the difficulties of research work concerning global exponential synchronization of periodic solution of complex BAM neural networks with time-varying and discontinuous activations.

Motivated by the above discussions, in this paper, we consider the complex BAM neural networks with periodic coefficients and discontinuous activations:

$$
\left\{\begin{array}{l}
\frac{d x_{i}(t)}{d t}=-a_{i}(t) x_{i}(t)+\sum_{j=1}^{m} p_{j i}(t) f_{j}\left(y_{j}(t)\right)+\sum_{j=1}^{m} q_{j i}(t) g_{j}\left(y_{j}(t-\tau(t))\right)+c_{i}(t), i=1,2, \ldots, n, \\
\frac{d y_{j}(t)}{d t}=-b_{j}(t) y_{j}(t)+\sum_{i=1}^{n} r_{i j}(t) u_{i}\left(x_{i}(t)\right)+\sum_{i=1}^{n} s_{i j}(t) v_{i}\left(x_{i}(t-\tau(t))\right)+d_{j}(t), j=1,2, \ldots, m,
\end{array}\right.
$$

where $n, m$ correspond to the number of units in a neural network, $x_{i}(t)$ denotes the state variable associated with the $i$ th neuron, $y_{j}(t)$ denotes the state variable associated with the $j$ th neuron; $a_{i}(t)>0$, $b_{i}(t)>0$, they denote the neural self-inhibitions; $p_{j i}(t), r_{i j}(t)$ and $q_{j i}(t), s_{i j}(t)$ denote the strengths of the $j$ th unit on the $i$ th unit at time $t$ and time $t-\tau(t)$, respectively; $c_{i}(t), d_{j}(t)$ are continuous $\omega$-periodic functions which denote the external inputs from outside the neural networks at time $t ; f_{j}, g_{j}$ and $u_{i}, v_{i}$ represent the neuron input-output activations; $\tau(t)$ corresponds to the transmission delay and satisfies $0 \leqslant \tau(t) \leqslant \tau\left(\tau=\max _{0 \leqslant t \leqslant \omega}\{\tau(t)\}\right.$ is a positive constant) and $\dot{\tau}(t) \leqslant \tau^{D}<1$. Throughout this paper, we always assume that $a_{i}(t), b_{i}(t), p_{j i}(t), r_{i j}(t), q_{i j}(t), s_{i j}(t), c_{i}(t), d_{j}(t)$ are continuous $w$-periodic functions.

$$
\begin{aligned}
& \text { Set } z(t)=\left(z_{1}(t), \ldots, z_{n}(t), z_{n+1}(t), \ldots, z_{n+m}(t)\right)^{\prime}=\left(x_{1}(t), \ldots, x_{n}(t), y_{1}(t), \ldots, y_{m}(t)\right)^{\prime}, I(t)= \\
& \left(c_{1}(t), \ldots, c_{n}(t), d_{1}(t), \ldots, d_{m}(t)\right)^{\prime}, \quad h(z(t))=\left(u_{1}\left(x_{1}(t)\right), \ldots, u_{n}\left(x_{n}(t)\right), f_{1}\left(y_{1}(t)\right), \ldots, f_{m}\left(y_{m}(t)\right)\right)^{\prime}, \\
& P=\left(p_{i j}(t)\right)_{m \times n}, Q=\left(q_{i j}(t)\right)_{m \times n}, \widetilde{h}(z(t-\tau(t)))=\left(v_{1}\left(x_{1}(t-\tau(t))\right), \ldots, v_{n}\left(x_{n}(t-\tau(t))\right), g_{1}\left(y_{1}(t-\right.\right. \\
& \left.\tau(t))), \ldots, g_{m}\left(y_{m}(t-\tau(t))\right)\right)^{\prime}, R=\left(r_{j i}(t)\right)_{n \times m}, S=\left(s_{j i}(t)\right)_{n \times m}, \text { and } A=\left(\begin{array}{l}
P^{\prime} \\
R^{\prime}
\end{array}\right), B=\left(\begin{array}{l}
Q^{\prime} \\
S^{\prime}
\end{array}\right), \\
& D=\operatorname{diag}\left(a_{1}(t), \ldots, a_{n}(t), b_{1}(t), \ldots, b_{m}(t)\right) .
\end{aligned}
$$

Eq. (1.1) can be equivalently represented by

$$
\frac{\mathrm{d} z(\mathrm{t})}{\mathrm{dt}}=-\mathrm{D} z(\mathrm{t})+\mathrm{Ah}(z(\mathrm{t}))+\mathrm{B} \widetilde{h}(z(t-\tau(t)))+\mathrm{I}(\mathrm{t}) .
$$


In order to establish our main results, it is necessary to give the following condition for the neuron activation functions in system (1.1) (or (1.2)):

( $(1)$ For every $i=1,2, \ldots, n, j=1,2, \ldots, m, f_{j}, g_{j}$ and $u_{i}, v_{i}$ are continuous in $\mathbb{R}$ except a countable set of isolate jump discontinuous points $\rho_{k}$, where there exist finite right and left limits, and in every compact set of $\mathbb{R}$, has only a finite number of jump discontinuous points.

Given a set $X \subset \mathbb{R}^{n}$, by $\overline{c o}[X]$ we denote the closure of the convex hull of $X$, and $\mu(X)$ denotes the Lebesgue measure in $\mathbb{R}^{n}$ of $X$. By the assumption ( $\mathbf{X} 1$, we can obtain:

$$
\begin{aligned}
& \overline{\mathrm{co}}[\mathrm{h}(z)]=\left(\overline{\mathrm{co}}\left[\mathrm{u}_{1}\left(x_{1}\right)\right], \ldots, \overline{\mathrm{co}}\left[\mathrm{u}_{\mathfrak{n}}\left(x_{\mathfrak{n}}\right)\right], \overline{\mathrm{co}}\left[\mathrm{f}_{1}\left(\mathrm{y}_{1}\right)\right], \ldots, \overline{\mathrm{co}}\left[\mathrm{f}_{\mathrm{m}}\left(\mathrm{y}_{\mathrm{m}}\right)\right]\right), \\
& \overline{\mathrm{co}}[\widetilde{\mathrm{h}}(z)]=\left(\overline{\mathrm{co}}\left[v_{1}\left(x_{1}\right)\right], \ldots, \overline{\mathrm{co}}\left[v_{\mathfrak{n}}\left(x_{n}\right)\right], \overline{\mathrm{co}}\left[\mathrm{g}_{1}\left(\mathrm{y}_{1}\right)\right], \ldots, \overline{\mathrm{co}}\left[\mathrm{g}_{\mathrm{m}}\left(\mathrm{y}_{\mathrm{m}}\right)\right]\right),
\end{aligned}
$$

where $\overline{\operatorname{co}}\left[u_{\mathfrak{i}}\left(x_{i}\right)\right]=\left[\min \left\{u_{i}\left(x_{i}^{-}\right), u_{i}\left(x_{i}^{+}\right)\right\}, \max \left\{u_{i}\left(x_{i}^{-}\right), u_{i}\left(x_{i}^{+}\right)\right\}\right], \overline{c o}\left[f_{j}\left(y_{j}\right)\right]=\left[\min \left\{f_{j}\left(y_{j}^{-}\right), f_{j}\left(y_{j}^{+}\right)\right\}, \max \{\right.$ $\left.\left.f_{j}\left(y_{j}^{-}\right), f_{j}\left(y_{j}^{+}\right)\right\}\right], \overline{c o}\left[v_{i}\left(x_{i}\right)\right]=\left[\min \left\{v_{i}\left(x_{i}^{-}\right), v_{i}\left(x_{i}^{+}\right)\right\}, \max \left\{v_{i}\left(x_{i}^{-}\right), v_{i}\left(x_{i}^{+}\right)\right\}\right], \overline{c o}\left[g_{j}\left(y_{j}\right)\right]=\left[\min \left\{g_{j}\left(y_{j}^{-}\right), g_{j}\left(y_{j}^{+}\right)\right\}\right.$, $\left.\max \left\{g_{j}\left(y_{j}^{-}\right), g_{j}\left(y_{j}^{+}\right)\right\}\right], i=1,2, \ldots, n, j=1,2, \ldots, m$. There exist measurable functions $\gamma=\left(\gamma_{1}, \ldots, \gamma_{n}\right.$, $\left.\gamma_{n+1}, \ldots, \gamma_{n+m}\right)^{\prime}$ such that $\gamma_{i} \in \overline{c o}\left[u_{i}\left(x_{i}\right)\right], i=1, \ldots, n$ and $\gamma_{n+j} \in \overline{c o}\left[f_{j}\left(y_{j}\right)\right], j=1, \ldots, m$, measurable functions $\eta=\left(\eta_{1}, \ldots, \eta_{n}, \eta_{n+1}, \ldots, \eta_{n+m}\right)^{\prime}$ such that $\eta_{i} \in \overline{c o}\left[v_{i}\left(x_{i}\right)\right], i=1, \ldots, n$ and $\eta_{n+j} \in \overline{c o}\left[g_{j}\left(y_{j}\right)\right]$, $j=1, \ldots, m$.

( $\aleph 2)$ For every $i \in \mathbb{N}$, there exist nonnegative constants $\alpha_{i}$ and $\beta_{i}$ such that

$$
\begin{gathered}
\sup _{\gamma_{i} \in \overline{\operatorname{co}}\left[h_{i}\left(z_{i}\right)\right]}\left|\gamma_{i}\right| \leqslant \alpha_{i}\left|z_{i}\right|+\beta_{i}, \sup _{\eta_{i} \in \overline{\operatorname{co}}\left[\tilde{h}_{i}\left(z_{i}\right)\right]}\left|\eta_{i}\right| \leqslant \alpha_{i}\left|z_{i}\right|+\beta_{i}, \forall z_{i} \in \mathbb{R}, \\
\sup _{\gamma_{i} \in \overline{c o}\left[h_{i}\left(z_{i}\right)\right]}\left|\gamma_{i}\right| \leqslant \alpha_{i}\left|z_{i}\right|+\beta_{i}, \sup _{\eta_{i} \in \overline{\operatorname{co}}\left[\tilde{h}_{i}\left(z_{i}\right)\right]}\left|\eta_{i}\right| \leqslant \alpha_{i}\left|z_{i}\right|+\beta_{i}, \forall z_{i} \in \mathbb{R} .
\end{gathered}
$$

The purpose of this paper is to realize the global exponential synchronization of complex discontinuous time-delayed BAM network system using the fixed point theorem of differential inclusion theory and by stabilization control. In the next section, some basic definitions and preliminary lemmas are introduced. In Section 3, the proof on the existence of periodic solution is presented. In Section 4, global exponential synchronization stability of periodic solution is obtained. In Section 5, we give a numerical example to illustrate our theoretical results and the proposed methods. Finally, we conclude this paper in Section 6.

In the following we introduce some notations which will be used in the rest of paper.

Let $\mathbb{R}^{n}$ denote the $n$-dimensional Euclidean space and the superscript $T$ denote the transposition. Let $x=\left(x_{1}, x_{2}, \ldots, x_{n}\right)^{\prime}, y=\left(y_{1}, y_{2}, \ldots, y_{n}\right)^{\prime}$, by $x>0(x \geqslant 0)$ we mean that $x_{i}>0\left(x_{i} \geqslant 0\right)$ for all $i=1,2, \ldots, n .\|x\|=\left(\sum_{i=1}^{n} x_{i}^{2}\right)^{\frac{1}{2}}$ denotes Euclidean norm of $x .\langle x, y\rangle=\sum_{i=1}^{n} x_{i} y_{i},\langle\cdot, \cdot\rangle$ denotes inner product. If $x \in \mathbb{R},\|x\|$ denotes any vector norm of $x$, while $\|x\|_{1}=\sum_{i=1}^{n}\left|x_{i}\right|$ and $\|x\|_{2}=\left(\sum_{i=1}^{n} x_{i}^{2}\right)^{1 / 2}$. Given real matrix $A=\left(a_{i j}\right)_{n \times n}, \lambda_{\max }(A)$ and $\lambda_{\min }(A)$ represent the maximal and minimal eigenvalues of $A$, respectively. Let $\operatorname{diag}(\cdots)$ denote a block-diagonal matrix and $\operatorname{sign}(\cdot)$ denote the sign function. Finally, for any continuous $\omega$-periodic function $f(t)$ defined on $\mathbb{R}$, we set

$$
\bar{f}=\frac{1}{\omega} \int_{0}^{\omega} f(t) d t, \quad f^{M}=\sup _{t \in[0, \omega]}|f(t)|, \quad f^{L}=\inf _{t \in[0, w]}|f(t)| .
$$

\section{Preliminaries}

In this section, we introduce some lemmas and definitions about set-valued analysis, differential inclusions, and non-smooth analysis (see $[1,3,8,13]$ ), which will be used throughout this paper.

Let $\mathbb{R}^{n}(n \geqslant 1)$ be an $n$-dimensional real Euclidean space with inner product $\langle\cdot, \cdot\rangle$ and induced norm $\|\cdot\|$. Suppose $X \subseteq \mathbb{R}^{n}$, let us denote

$$
P_{0}(X) \triangleq 2^{X}=\{A \subset X: A \neq \varnothing\}, P(x)=P_{0} \cup\{\varnothing\},
$$




$$
\begin{aligned}
& P_{f(c)}(X)=\{A \subset X:(\text { convex }) \text { nonempty and closed }\}, \\
& P_{k(c)}(X)=\{A \subset X:(\text { convex }) \text { nonempty and compact }\} .
\end{aligned}
$$

For given $A \subset P_{f}(X), x \in X$, the distance from $x$ to $A$ is defined as $\operatorname{dist}(x, A)=\inf \{\|x-a\|: a \in A\}$. Let $K\left(\mathbb{R}_{n}\right)$ denote the collection of all nonempty compact subsets of $\mathbb{R}^{n}$ with the Hausdorff metric $\rho$ defined by

$$
\rho(A, B)=\max \{\beta(A, B), \beta(B, A)\}, \quad A, B \in K\left(\mathbb{R}^{n}\right),
$$

where

$$
\beta(A, B)=\sup \{\operatorname{dist}(x, B)\}, \quad \beta(B, A)=\sup \{\operatorname{dist}(y, A)\} .
$$

Obviously, with metric $\rho, K\left(\mathbb{R}^{\mathfrak{n}}\right)$ is a complete metric space. Let

$$
\mathrm{K} v\left(\mathbb{R}^{\mathrm{n}}\right)=\left\{\mathrm{A} \in \mathrm{K}\left(\mathbb{R}^{\mathrm{n}}\right) \mathrm{A} \text { is convex }\right\} .
$$

Now we introduce the concept to Filippov solution (see [10]). Consider the following differential system in vector notation:

$$
\frac{d x}{d t}=f(t, x)
$$

where $f(t, x)$ is discontinuous in $x$.

Definition $2.1([10,14])$. Suppose $E \subset \mathbb{R}^{n}$. Then $x \mapsto F(x)$ is called a set-valued map from $E \hookrightarrow \mathbb{R}^{n}$ if to each point $x$ of a set $E \subset \mathbb{R}^{n}$, there corresponds a nonempty set $F(x) \subset \mathbb{R}^{n}$. A set-valued map $\mathrm{F}: \mathrm{E} \rightarrow \mathrm{K}\left(\mathbb{R}^{n}\right)$ is said to be upper semicontinuous (USC) at $x_{0} \in E$, if $\beta\left(F(x), F\left(x_{0}\right)\right) \rightarrow 0$ as $x \rightarrow x_{0}$. $F(x)$ is said to have a closed (convex, compact) image if for each $x \in E, F(x)$ is closed (convex, compact). $\operatorname{Graph}(F(E))=\{(x, y): x \in E$, and $y \in F(x)\}$, where $E$ is subset of $\mathbb{R}^{n}$.

Definition $2.2([10,14])$. Consider the autonomous system $\frac{d x}{d t}=f(x)$, where $f(x)$ is discontinuous in $x \in \mathbb{R}^{n}$. Let us construct a Filippov set-valued map (i.e., Filippov regularization) $\mathrm{F}: \mathbb{R}^{n} \times \mathbb{C} \rightarrow 2^{\mathbb{R}^{n}}$ defined as

$$
F(x)=\bigcap_{\delta>0} \bigcap_{\mu(\mathcal{N})=0} \overline{\operatorname{co}}[f(\mathfrak{B}(x, \delta) \backslash \mathcal{N})] .
$$

where $\mu(\mathcal{N})$ denotes the Lebesgue measure of set $\mathcal{N}$; intersection is taken over all sets $\mathcal{N}$ of Lebesgue measure zero and over all $\delta>0 ; \mathfrak{B}(x, \delta):=\left\{y \in \mathbb{R}^{\mathfrak{n}}:\|y-x\| \leqslant \delta\right\}$ represents the ball of center $x$ and radius $\delta ; \overline{c o}[\mathbb{E}]$ is the closure of the convex hull of set $\mathbb{E}$. A vector-valued function $x(t)$ defined on a nondegenerate interval $\mathcal{J} \in \mathbb{R}$ is said to be a Filippov solution of this discontinuous system, if it is absolutely continuous on any compact subinterval $\left[t_{1}, t_{2}\right]$ of $\mathcal{J}$, and for almost all $t \in \mathcal{J}, x(t)$ satisfies the differential inclusion

$$
\frac{d x}{d t} \in F(t, x) \text {, for a.e. } t \in \mathcal{J} \text {. }
$$

By Definition 2.2, we can get the definition of the Filippov solution of (1.2) as follows:

A vector function $z(t)=\left(x_{1}(t), \ldots, x_{n}(t), y_{1}(t), \ldots, y_{m}(t)\right)^{\prime}:[-\tau, T) \rightarrow \mathbb{R}^{n+m}, T \in(0, \infty]$, is a state solution of the delayed and discontinuous system (1.1) on $[-\tau, T)$, if $z(t)$ is absolutely continuous on any compact interval of $[-\tau, T)$ and satisfies differential inclusion

$$
\frac{\mathrm{d} z(\mathrm{t})}{\mathrm{dt}} \in-\mathrm{D} z(\mathrm{t})+A \overline{c o}[\mathrm{~h}(z(\mathrm{t}))]+\mathrm{B} \overline{\mathrm{co}}[\widetilde{\mathrm{h}}(z(\mathrm{t}-\tau(\mathrm{t})))]+\mathrm{I}(\mathrm{t}) .
$$

Obviously, the set-valued map $\phi(z, t):(z(t), t) \hookrightarrow-D z(t)+A \overline{c o}[h(z(t))]+B \overline{c o}[\widetilde{h}(z(t-\tau(t)))]+I(t)$ has nonempty compact convex values, moreover, it is upper semicontinuous. Hence, it is measurable. By 
measurable selection theorem, we get that if there exist bounded measurable functions $\gamma(t) \in \overline{c o}[h(z(t))]$ and $\eta(t-\tau(t)) \in \overline{c o}[\widetilde{h}(z(t-\tau(t)))]$ such that

$$
\frac{\mathrm{d} z(\mathrm{t})}{\mathrm{dt}}=-\mathrm{D} z(\mathrm{t})+\mathrm{A} \gamma(\mathrm{t})+\mathrm{B \eta}(\mathrm{t}-\tau(\mathrm{t}))+\mathrm{I}(\mathrm{t}),
$$

then $z(t)$ is a solution of Eq. (1.2).

The lemma below is the set-valued version of the Mawhin coincidence theorem on the existence of periodic solution and its proof can be found in [21].

Lemma 2.3 ([21]). Suppose that $\mathrm{F}: \mathbb{R} \times \mathbb{R}^{n} \rightarrow \mathrm{K} v\left(\mathbb{R}^{\mathrm{n}}\right)$ is USC and w-periodic in $\mathrm{t}$. If the following conditions hold:

(1) there exists a bounded open set $\Omega \subseteq \mathrm{C}_{\omega}$, the set of all continuous, $\omega$-periodic functions: $\mathbb{R} \rightarrow \mathbb{R}^{n}$, such that for any $\lambda \in(0,1)$, each $\omega$-periodic function $x(t)$ of the inclusion

$$
\frac{d x}{d t} \in \lambda F(t, x)
$$

satisfies $x \notin \partial \Omega$ if it exists;

(2) each solution $\mathrm{u} \in \mathbb{R}^{\mathrm{n}}$ of the inclusion

$$
0 \in \frac{1}{\omega} \int_{0}^{\omega} F(t, u) d t=g_{0}(u)
$$

satisfies $u \notin \partial \Omega \cap \mathbb{R}^{n}$;

(3) $\operatorname{deg}\left(g_{0}, \Omega \cap \mathbb{R}^{n}, 0\right) \neq 0$,

then differential inclusion (2.1) has at least one w-periodic solution $x(t)$ with $x \in \bar{\Omega}$.

Definition 2.4 ([7]). We say that real matrix $\Theta=\left(\theta_{i j}\right) \in \mathbb{R}^{n \times n}$ is an M-matrix, if and only if we have $\theta_{i j} \leqslant 0, i, j=1,2, \ldots, n, i \neq j$, and all successive principal minors of $\Theta$ are positive.

Lemma 2.5 ([2,18]). Let $\Theta=\left(\theta_{i j}\right)$ be an $\mathrm{n} \times \mathrm{n}$ matrix with non-positive off-diagonal elements. The $\Theta$ is an M-matrix if and only if one of the following conditions holds:

(1) there exists a vector $\xi=\left(\xi_{1}, \xi_{2}, \ldots, \xi_{n}\right)>(0,0, \ldots, 0)$ such that $\xi \Theta>0$;

(2) there exists a vector $\eta=\left(\eta_{1}, \eta_{2}, \ldots, \eta_{n}\right)^{\top}>(0,0, \ldots, 0)^{\top}$ such that $\Theta \eta>0$.

Lemma 2.6 ([11, 12]). If $\mathrm{V}(\mathrm{x}): \mathbb{R}^{\mathrm{n}} \rightarrow \mathbb{R}$ is C-regular, and $x(\mathrm{t}):[0,+\infty) \rightarrow \mathbb{R}^{\mathrm{n}}$ is absolutely continuous on any compact subinterval of $[0,+\infty)$, then, $x(t)$ and $\mathrm{V}(\mathrm{x}(\mathrm{t})):[0,+\infty) \rightarrow \mathbb{R}$ are differential for almost all $\mathrm{t} \in[0,+\infty)$ and

$$
\frac{\mathrm{dV}(x(\mathrm{t}))}{\mathrm{dt}}=\left\langle\sigma(\mathrm{t}), \frac{\mathrm{d} x(\mathrm{t})}{\mathrm{dt}}\right\rangle, \forall \sigma(\mathrm{t}) \in \partial \mathrm{V}(x(\mathrm{t}))
$$

\section{Existence of the periodic solution}

In this section, we will explore the existence of periodic solution for system (1.1) (or (1.2)) with discontinuous activation functions. Our main tools to be used involve the application of M-matrix theory, differential inclusions theory, and set-valued analysis.

First of all, define

$$
\mathrm{C}_{\omega}=\left\{x(\mathrm{t}) \in \mathrm{C}\left(\mathbb{R}, \mathbb{R}^{\mathrm{n}+\mathrm{m}}\right): x(\mathrm{t}+\omega)=x(\mathrm{t})\right\}, \quad\|x(\mathrm{t})\|_{\mathrm{C}_{\omega}}=\sum_{i=1}^{\mathrm{n}+\mathrm{m}} \max _{\mathrm{t} \in[0, \omega)}\left|x_{i}(\mathrm{t})\right| .
$$


Then $C_{\omega}$ is a Banach space with norm $\|\cdot\|_{C_{\omega}}$. Let for $z(t)=\left(x_{1}(t), \ldots, x_{n}(t), y_{1}(t), \ldots, y_{m}(t)\right)^{\prime} \in C_{\omega}$, $F(t, z)=\left(F_{1}(t, x), \ldots, F_{n}(t, x), F_{n+1}(t, y), \ldots, F_{n+m}(t, y)\right)^{\prime}$, where

$$
\begin{aligned}
F_{i}(t, x) & =-a_{i}(t) x_{i}(t)+\sum_{j=1}^{m} p_{j i}(t) \overline{c o}\left[f_{j}\left(y_{j}(t)\right)\right]+\sum_{j=1}^{m} q_{j i}(t) \overline{c o}\left[g_{j}\left(y_{j}(t-\tau(t))\right)\right]+c_{i}(t), i=1, \ldots, n, \\
F_{n+j}(t, y) & =-b_{j}(t) y_{j}(t)+\sum_{i=1}^{n} r_{i j}(t) \overline{c o}\left[u_{i}\left(x_{i}(t)\right)\right]+\sum_{i=1}^{n} s_{i j}(t) \overline{c o}\left[v_{i}\left(x_{i}(t-\tau(t))\right)\right]+d_{j}(t), j=1, \ldots, m .
\end{aligned}
$$

In order to conduct with our analysis on periodic solutions, we give the following assumption:

(メ3) $E-M$ is an M-matrix, where $M=\left(m_{i j}\right)_{(n+m) \times(n+m)}=\left(\begin{array}{ll}M_{2}^{\prime} & M_{1}\end{array}\right)$, and

$$
M_{1}=\left(\frac{\alpha_{n+j}}{a_{i}^{L}}\left(p_{j i}^{M}+\frac{q_{j i}^{M}}{\sqrt{1-\tau^{D}}}\right)\right)_{n \times m}, M_{2}=\left(\frac{\alpha_{i}}{b_{j}^{L}}\left(r_{i j}^{M}+\frac{s_{i j}^{M}}{\sqrt{1-\tau^{D}}}\right)\right)_{n \times m}, i=1,2, \ldots, n, j=1,2, \ldots, m .
$$

Then, we will give some propositions, which are important tools to proceed the existence of $\omega$-periodic solution to the discontinuous network system (1.1) (or (1.2)).

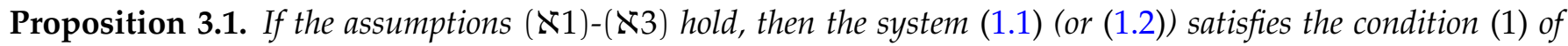
Lemma 2.3.

Proof. Set $h(z(t))=\left(h_{1}\left(z_{1}(t)\right), \ldots, h_{n}\left(z_{n}(t)\right), h_{n+1}\left(z_{n+1}(t)\right), \ldots, h_{n+m}\left(z_{n+m}(t)\right)\right)^{\prime}=\left(u_{1}\left(z_{1}(t)\right), \ldots, u_{n}(\right.$ $\left.\left.z_{\mathfrak{n}}(t)\right), f_{1}\left(z_{\mathfrak{n}+1}(t)\right), \ldots, f_{m}\left(z_{n+m}(t)\right)\right)^{\prime}, \widetilde{h}(z(t-\tau(t)))=\left(\widetilde{h}_{1}\left(z_{1}(t-\tau(t))\right), \ldots, \widetilde{h}_{\mathfrak{n}}\left(z_{\mathfrak{n}}(t-\tau(t))\right), \widetilde{h}_{n+1}\left(z_{n+1}(t-\right.\right.$ $\left.\tau(t))), \ldots, \widetilde{h}_{n+m}\left(z_{n+m}(t-\tau(t))\right)\right)^{\prime}=\left(v_{1}\left(z_{1}(t-\tau(t))\right), \ldots, v_{n}\left(z_{n}(t-\tau(t))\right), g_{1}\left(z_{n+1}(t-\tau(t))\right), \ldots, g_{m}(\right.$ $\left.\left.z_{n+m}(t-\tau(t))\right)\right)^{\prime}$. It is clear that $F(t, z)$ is an USC set-valued map with nonempty compact convex values. In order to apply the condition (1) of Lemma 2.3, we need to search for appropriate open, bounded subset $\Omega$. Corresponding to the differential inclusion $\frac{d z}{d t} \in \lambda F(z, t), \lambda \in(0,1)$, we have

$$
\left\{\begin{aligned}
\frac{d x_{i}(t)}{d t} \in \lambda & {\left[-a_{i}(t) x_{i}(t)+\sum_{j=1}^{m} p_{j i}(t) \overline{c o}\left[h_{n+j}\left(z_{n+j}(t)\right)\right]\right.} \\
& \left.+\sum_{j=1}^{m} q_{j i}(t) \overline{c o}\left[\widetilde{h}_{n+j}\left(z_{n+j}(t-\tau(t))\right)\right]+c_{i}(t)\right], i=1,2, \ldots, n, \\
\frac{d y_{j}(t)}{d t} \in & \lambda\left[-b_{j}(t) y_{j}(t)+\sum_{i=1}^{n} r_{i j}(t) \overline{c o}\left[h_{i}\left(z_{i}(t)\right)\right]\right. \\
& \left.+\sum_{i=1}^{n} s_{i j}(t) \overline{c o}\left[\widetilde{h}_{i}\left(z_{i}(t-\tau(t))\right)\right]+d_{j}(t)\right], j=1,2, \ldots, m,
\end{aligned}\right.
$$

assume that $z(t)=\left(x_{1}(t), \ldots, x_{n}(t), y_{1}(t), \ldots, y_{m}(t)\right)^{\prime}$ is an arbitrary $\omega$-periodic solution of the differential inclusion (3.1) for a certain $\lambda \in(0,1)$. By the measurable selection theorem, we can find the measurable function $\gamma=\left(\gamma_{1}, \ldots, \gamma_{n}, \gamma_{n+1}, \ldots, \gamma_{n+m}\right)^{\prime}$ and $\eta=\left(\eta_{1}, \ldots, \eta_{n}, \eta_{n+1}, \ldots, \eta_{n+m}\right)^{\prime}$ such that $\gamma_{j}(t) \in \overline{c o}\left[h_{j}\left(z_{j}(t)\right)\right], j=1,2, \ldots, n+m$, and $\eta_{j}(t) \in \overline{c o}\left[\widetilde{h}_{j}\left(z_{j}(t)\right)\right], j=1,2, \ldots, n+m$, for a.e. $t \in[-\tau, b)$ and

$$
\left\{\begin{array}{l}
\frac{d x_{i}(t)}{d t}=\lambda\left[-a_{i}(t) x_{i}(t)+\sum_{j=1}^{m} p_{j i}(t) \gamma_{n+j}(t)+\sum_{j=1}^{m} q_{j i}(t) \eta_{n+j}(t-\tau(t))+c_{i}(t)\right], i=1,2, \ldots, n, \\
\frac{d y_{j}(t)}{d t}=\lambda\left[-b_{j}(t) y_{j}(t)+\sum_{i=1}^{n} r_{i j}(t) \gamma_{i}(t)+\sum_{i=1}^{n} s_{i j}(t) \eta_{i}(t-\tau(t))+d_{j}(t)\right], j=1,2, \ldots, m .
\end{array}\right.
$$


Multiplying both sides of the first equation of (3.2) by $x_{\mathfrak{i}}(t)$ and integrating over the interval $[0, \omega]$, we obtain

$$
\int_{0}^{\omega} a_{i}(t) x_{i}^{2}(t) d t=\int_{0}^{\omega} x_{i}(t)\left[\sum_{j=1}^{m} p_{j i}(t) \gamma_{n+j}(t)+\sum_{j=1}^{m} q_{j i}(t) \eta_{n+j}(t-\tau(t))+c_{i}(t)\right] d t .
$$

In view of $(\aleph 2)$ and Cauchy-Schwarz inequality, we derive from (3.3) that

$$
\begin{aligned}
a_{i}^{L} \int_{0}^{\omega}\left|x_{i}(t)\right|^{2} d t \leqslant & \sum_{j=1}^{m} p_{j i}^{M} \int_{0}^{\omega}\left|x_{i}(t)\right|\left|\gamma_{n+j}(t)\right| d t \\
& +\sum_{j=1}^{m} q_{j i}^{M} \int_{0}^{\omega}\left|x_{i}(t)\right|\left|\eta_{n+j}(t-\tau(t))\right| d t+c_{i}^{M} \int_{0}^{\omega}\left|x_{i}(t)\right| d t \\
\leqslant & \sum_{j=1}^{m} p_{j i}^{M} \alpha_{n+j} \int_{0}^{\omega}\left|x_{i}(t)\right|\left|y_{j}(t)\right| d t+\sum_{j=1}^{m} q_{j i}^{M} \alpha_{n+j} \int_{0}^{\omega}\left|x_{i}(t)\right|\left|y_{j}(t-\tau(t))\right| d t \\
& +\left(\sum_{j=1}^{m} p_{j i}^{M} \beta_{n+j}+\sum_{j=1}^{m} q_{j i}^{M} \beta_{n+j}+c_{i}^{M}\right) \int_{0}^{\omega}\left|x_{i}(t)\right| d t \\
\leqslant & \sum_{j=1}^{m} p_{j i}^{M} \alpha_{n+j}\left(\int_{0}^{\omega}\left|x_{i}(t)\right|^{2} d t\right)^{1 / 2} \times\left(\int_{0}^{\omega}\left|y_{j}(t)\right|^{2} d t\right)^{1 / 2} \\
& +\sum_{j=1}^{m} q_{j i}^{M} \alpha_{n+j}\left(\int_{0}^{\omega}\left|x_{i}(t)\right|^{2} d t\right)^{1 / 2} \times\left(\int_{0}^{\omega}\left|y_{j}(t-\tau(t))\right|^{2} d t\right)^{1 / 2} \\
& +\left(\sum_{j=1}^{m} p_{j i}^{M} \beta_{n+j}+\sum_{j=1}^{m} q_{j i}^{M} \beta_{n+j}+c_{i}^{M}\right) \sqrt{\omega} \times\left(\int_{0}^{\omega}\left|x_{i}(t)\right|^{2} d t\right)^{1 / 2},
\end{aligned}
$$

we notice that

$$
\begin{aligned}
\int_{0}^{\omega}\left|y_{j}(t-\tau(t))\right|^{2} d t & =\int_{-\tau(0)}^{\omega-\tau(w)} \frac{\left|y_{j}(t)\right|^{2}}{1-\dot{\tau}\left(\rho^{-1}(t)\right)} d t=\int_{-\tau(0)}^{\omega-\tau(0)} \frac{\left|y_{j}(t)\right|^{2}}{1-\dot{\tau}\left(\rho^{-1}(t)\right)} d t \\
& =\int_{0}^{\omega} \frac{\left|y_{j}(t)\right|^{2}}{1-\dot{\tau}\left(\rho^{-1}(t)\right)} d t \leqslant \frac{1}{1-\tau^{D}} \int_{0}^{\omega}\left|y_{j}(t)\right|^{2} d t
\end{aligned}
$$

where $\rho^{-1}$ is the inverse function of $\rho(t)=t-\tau(t)$, then from (3.4) and (3.5), it implies

$$
\begin{aligned}
& \left(\int_{0}^{\omega}\left|x_{i}(t)\right|^{2} d t\right)^{1 / 2} \\
& \leqslant \sum_{j=1}^{m} \frac{\alpha_{n+j}}{a_{i}^{L}}\left(p_{j i}^{M}+\frac{q_{j i}^{M}}{\sqrt{1-\tau^{D}}}\right)\left(\int_{0}^{\omega}\left|y_{j}(t)\right|^{2} d t\right)^{1 / 2}+\frac{\sqrt{\omega}}{a_{i}^{L}}\left(\sum_{j=1}^{m}\left(p_{j i}^{M}+q_{j i}^{M}\right) \beta_{n+j}+c_{i}^{M}\right) \\
& \triangleq \sum_{j=1}^{m} \frac{\alpha_{n+j}}{a_{i}^{L}}\left(p_{j i}^{M}+\frac{q_{j i}^{M}}{\sqrt{1-\tau^{D}}}\right)\left(\int_{0}^{\omega}\left|y_{j}(t)\right|^{2} d t\right)^{1 / 2}+\sqrt{\omega} G_{i}^{\prime}, i=1,2, \ldots, n .
\end{aligned}
$$

where

$$
M_{1}=\left(\frac{\alpha_{n+j}}{a_{i}^{L}}\left(p_{j i}^{M}+\frac{q_{j i}^{M}}{\sqrt{1-\tau^{D}}}\right)\right)_{n \times m} \quad \text { and } \quad G_{i}^{\prime}=\frac{1}{a_{i}^{L}}\left(\sum_{j=1}^{m}\left(p_{j i}^{M}+q_{j i}^{M}\right) \beta_{n+j}+c_{i}^{M}\right) .
$$

Without loss of generality, multiplying both sides of the second equation of (3.2) by $y_{j}(t)$ and integrating 
over the interval $[0, \omega]$, it is easy to calculate that

$$
\begin{aligned}
\left(\int_{0}^{\omega}\left|y_{j}(t)\right|^{2} d t\right)^{1 / 2} & \leqslant \sum_{i=1}^{n} \frac{\alpha_{i}}{b_{j}^{L}}\left(r_{i j}^{M}+\frac{s_{i j}^{M}}{\sqrt{1-\tau^{D}}}\right)\left(\int_{0}^{\omega}\left|x_{i}(t)\right|^{2} d t\right)^{1 / 2}+\frac{\sqrt{\omega}}{b_{j}^{L}}\left(\sum_{i=1}^{n}\left(r_{i j}^{M}+s_{i j}^{M}\right) \beta_{i}+d_{j}^{M}\right) \\
& \triangleq \sum_{i=1}^{n} \frac{r_{i j}^{M} \alpha_{i}}{b_{j}^{L}}\left(\int_{0}^{\omega}\left|x_{i}(t)\right|^{2} d t\right)^{1 / 2}+\sqrt{\omega} G_{j}^{\prime \prime}, j=1,2, \ldots, m,
\end{aligned}
$$

where

$$
M_{2}=\left(\frac{\alpha_{i}}{b_{j}^{L}}\left(r_{i j}^{M}+\frac{s_{i j}^{M}}{\sqrt{1-\tau^{D}}}\right)\right)_{n \times m} \quad \text { and } G_{j}^{\prime \prime}=\frac{1}{b_{j}^{L}}\left(\sum_{i=1}^{n}\left(r_{i j}^{M}+s_{i j}^{M}\right) \beta_{i}+d_{j}^{M}\right) .
$$

Combining (3.6) and (3.7), we obtain

$$
\left(\int_{0}^{\omega}\left|z_{i}(t)\right|^{2} d t\right)^{1 / 2} \leqslant \sum_{j=1}^{n+m} m_{i j}\left(\int_{0}^{\omega}\left|z_{i}(t)\right|^{2} d t\right)^{1 / 2}+\sqrt{\omega} G_{i}, \quad i=1,2, \ldots, n+m,
$$

where

$$
G_{i}=G_{i}^{\prime} \text { when } i=1,2, \ldots, n \text {, and } G_{i}=G_{i-n}^{\prime \prime} \text { when } i=n+1, n+2, \ldots, n+m \text {. }
$$

For the sake of convenience, we define $\left\|z_{i}\right\|_{2}^{\omega}$ by

$$
\left\|z_{\mathfrak{i}}\right\|_{2}^{\omega}=\left(\int_{0}^{\omega}\left|z_{\mathfrak{i}}(\mathrm{t})\right|^{2} \mathrm{dt}\right)^{1 / 2}, z_{\mathfrak{i}} \in \mathrm{C}(\mathbb{R}, \mathbb{R}), \quad i=1,2, \ldots, n+m .
$$

Obviously, it follows from (3.8) that

$$
\begin{gathered}
\left(E_{n+m}-M\right)\left(\left\|z_{1}\right\|_{2}^{\omega}, \ldots,\left\|z_{n}\right\|_{2}^{\omega},\left\|z_{n+1}\right\|_{2}^{\omega}, \ldots,\left\|z_{n+m}\right\|_{2}^{\omega}\right)^{\prime} \\
\leqslant \sqrt{\omega}\left(G_{1}, \ldots, G_{n}, G_{n+1}, \ldots, G_{n+m}\right)^{\prime} \triangleq \sqrt{\omega} G .
\end{gathered}
$$

Since $E_{n+m}-M$ is an M-matrix, we can obtain from Lemma 2.5 and assumption ( $\times 3$ ) that there exists a vector $\xi=\left(\xi_{1}, \ldots, \xi_{n}, \xi_{n+1}, \ldots, \xi_{n+m}\right)>(0, \ldots, 0,0, \ldots, 0)$ such that

$$
\xi^{*}=\left(\xi_{1}^{*}, \ldots, \xi_{n}^{*}, \xi_{n+1}^{*}, \ldots, \xi_{n+m}^{*}\right)=\xi\left(E_{n+m}-M\right)>(0, \ldots, 0,0, \ldots, 0),
$$

together with (3.9), yields that

$$
\begin{aligned}
\min & \left\{\xi_{1}^{*}, \ldots, \xi_{n}^{*}, \xi_{n+1}^{*}, \ldots, \xi_{n+m}^{*}\right\}\left(\left\|z_{1}\right\|_{2}^{\omega}, \ldots,\left\|z_{n}\right\|_{2}^{\omega},\left\|z_{n+1}\right\|_{2}^{\omega}, \ldots,\left\|z_{n+m}\right\|_{2}^{\omega}\right) \\
& \leqslant \xi_{1}^{*}\left\|z_{1}\right\|_{2}^{\omega}+\ldots+\xi_{n}^{*}\left\|z_{n}\right\|_{2}^{\omega}+\ldots+\xi_{n+m}^{*}\left\|z_{n+m}\right\|_{2}^{\omega} \\
& =\xi\left(E_{n+m}-M\right)\left(\left\|z_{1}\right\|_{2}^{\omega}, \ldots,\left\|z_{n}\right\|_{2}^{\omega},\left\|z_{n+1}\right\|_{2}^{\omega}, \ldots,\left\|z_{n+m}\right\|_{2}^{\omega}\right)^{\prime} \\
& \leqslant \xi \sqrt{\omega}\left(G_{1}, \ldots, G_{n}, G_{n+1}, \ldots, G_{n+m}\right)^{\prime}=\sqrt{\omega} \sum_{i=1}^{n+m} \xi_{i} G_{i} .
\end{aligned}
$$

Thus, we have

$$
\left(\int_{0}^{\omega}\left|z_{i}(t)\right|^{2} d t\right)^{1 / 2}=\left\|z_{i}\right\|_{2}^{\omega} \leqslant \frac{\sqrt{\omega} \sum_{i=1}^{n+m} \xi_{i} G_{i}}{\min \left\{\xi_{1}^{*}, \ldots, \xi_{n}^{*}, \xi_{n+1}^{*}, \ldots, \xi_{n+m}^{*}\right\}} \triangleq \sqrt{\omega} N, \quad i=1,2, \ldots, n+m,
$$


where

$$
N=\frac{\sum_{i=1}^{n+m} \xi_{i} G_{i}}{\min \left\{\xi_{1}^{*}, \ldots, \xi_{n}^{*}, \xi_{n+1}^{*}, \ldots, \xi_{n+m}^{*}\right\}}, \quad i=1,2, \ldots, n+m
$$

It is easy to check that there exists $t_{i} \in[0, \omega]$ such that

$$
\left|z_{i}\left(t_{i}\right)\right| \leqslant N, \quad i=1,2, \ldots, n+m .
$$

Since for $t \in[0, \omega]$,

$$
z_{\mathfrak{i}}(\mathrm{t})=z_{\mathfrak{i}}\left(\mathrm{t}_{\mathrm{i}}\right)+z_{\mathfrak{i}}(\mathrm{t})-z_{\mathfrak{i}}\left(\mathrm{t}_{\mathrm{i}}\right)=z_{\mathfrak{i}}\left(\mathrm{t}_{\mathrm{i}}\right)+\int_{\mathrm{t}_{\mathfrak{i}}}^{\mathrm{t}} \dot{z}_{\mathfrak{i}}(\mathrm{s}) \mathrm{ds},
$$

it follows for (3.10) that

$$
\left|z_{\mathfrak{i}}(\mathrm{t})\right| \leqslant \mathrm{N}+\int_{0}^{\omega}\left|\dot{z}_{\mathfrak{i}}(\mathrm{t})\right| \mathrm{dt}, \quad i=1,2, \ldots, \mathrm{n}+\mathrm{m} .
$$

From (3.2), we obtain that

$$
\begin{aligned}
& \int_{0}^{\omega}\left|\dot{x}_{i}(t)\right| d t \\
& <\int_{0}^{\omega}\left|a_{i}(t)\left\|x_{i}(t)\left|d t+\sum_{j=1}^{m} \int_{0}^{\omega}\right| p_{j i}(t)\right\| \gamma_{n+j}(t)\right| d t \\
& +\sum_{j=1}^{m} \int_{0}^{\omega}\left|q_{j i}(t)\right|\left|\eta_{n+j}(t-\tau(t))\right| d t+\int_{0}^{\omega}\left|c_{i}(t)\right| d t \\
& \leqslant a_{i}^{M} \int_{0}^{\omega}\left|x_{i}(t)\right| d t+\sum_{j=1}^{m} p_{j i}^{M} \int_{0}^{\omega}\left|\gamma_{n+j}(t)\right| d t+\sum_{j=1}^{m} q_{j i}^{M} \int_{0}^{\omega}\left|\eta_{n+j}(t-\tau(t))\right| d t+c_{i}^{M} \omega \\
& \leqslant a_{i}^{M} \int_{0}^{\omega}\left|x_{i}(t)\right| d t+\sum_{j=1}^{m} p_{j i}^{M} \alpha_{n+j} \int_{0}^{\omega}\left|y_{j}(t)\right| d t+\sum_{j=1}^{m} q_{j i}^{M} \alpha_{n+j} \int_{0}^{\omega}\left|y_{j}(t-\tau(t))\right| d t \\
& +\omega\left(\sum_{j=1}^{m} p_{j i}^{M} \beta_{n+j}+\sum_{j=1}^{m} q_{j i}^{M} \beta_{n+j}+c_{i}^{M}\right) \\
& \leqslant a_{i}^{M} \sqrt{\omega}\left\|x_{i}\right\|_{2}^{\omega}+\sum_{j=1}^{m} \alpha_{n+j}\left(p_{j i}^{M}+\frac{q_{j i}^{M}}{\sqrt{1-\tau^{D}}}\right) \sqrt{\omega}\left\|y_{j}\right\|_{2}^{\omega}+\omega\left(\sum_{j=1}^{m} p_{j i}^{M} \beta_{n+j}+\sum_{j=1}^{m} q_{j i}^{M} \beta_{n+j}+c_{i}^{M}\right) \\
& \leqslant \omega N\left(a_{i}^{M}+\sum_{j=1}^{m} \alpha_{n+j}\left(p_{j i}^{M}+\frac{q_{j i}^{M}}{\sqrt{1-\tau^{D}}}\right)\right)+\omega\left(\sum_{j=1}^{m}\left(p_{j i}^{M}+q_{j i}^{M}\right) \beta_{n+j}+c_{i}^{M}\right) \triangleq R_{i}^{\prime}, i=1, \ldots, n .
\end{aligned}
$$

Similarly,

$$
\int_{0}^{\omega}\left|\dot{y}_{j}(t)\right| d t \leqslant \omega N\left(b_{j}^{M}+\sum_{i=1}^{n} \alpha_{i}\left(r_{i j}^{M}+\frac{s_{i j}^{M}}{\sqrt{1-\tau^{D}}}\right)\right)+\omega\left(\sum_{i=1}^{n}\left(r_{i j}^{M}+s_{i j}^{M}\right) \beta_{i}+d_{j}^{M}\right) \triangleq R_{j}^{\prime \prime}, j=1, \ldots, m .
$$

It follows from (3.12) and (3.13) that

$$
\int_{0}^{\omega}\left|\dot{z}_{i}(t)\right| d t \leqslant R_{i}, \quad i=1,2, \ldots, n+m,
$$


where

$$
R_{i}=R_{i}^{\prime} \text { when } i=1,2, \ldots, n \text {, and } R_{i}=R_{i-n}^{\prime \prime} \text { when } i=n+1, n+2, \ldots, n+m \text {. }
$$

By (3.11) and (3.14) we obtain that

$$
\left|z_{i}(t)\right|<N+R_{i} \triangleq H_{i}, \quad i=1,2, \ldots, n+m .
$$

Clearly, $H_{i}(i=1,2, \ldots, n+m)$ is independent of $\lambda$. Again from assumption ( $\left.\times 3\right)$ and Lemma 2.5, it follows that there exists a vector $\vartheta=\left(\vartheta_{1}, \ldots, \vartheta_{n}, \vartheta_{n+1}, \ldots, \vartheta_{n+m}\right)^{\prime}>(0, \ldots, 0,0, \ldots, 0)^{\prime}$ such that $\left(E_{n+m}-M\right) \vartheta>(0, \ldots, 0,0, \ldots, 0)^{\prime}$. Therefore, we can choose a sufficiently large constant $\sigma$ such that $\vartheta^{*}=\left(\vartheta_{1}^{*}, \ldots, \vartheta_{n}^{*}, \vartheta_{n+1}^{*}, \ldots, \vartheta_{n+m}^{*}\right)^{\prime}=\left(\sigma \vartheta_{1}, \ldots, \sigma \vartheta_{n}, \sigma \vartheta_{n+1}, \ldots, \sigma \vartheta_{n+m}\right)=\sigma \eta$ and

$$
\vartheta_{i}^{*}=\sigma \vartheta_{i}>H_{i}(i=1,2, \ldots, n+m) \text {, implies }\left(E_{n+m}-M\right) \vartheta^{*}>G \text {. }
$$

We take

$$
\Omega=\left\{z(\mathrm{t}) \in \mathrm{C}_{\omega} \mid-\vartheta^{*}<z(\mathrm{t})<\vartheta^{*}, \forall \mathrm{t} \in \mathbb{R}\right\} .
$$

Clearly $\Omega$ is an open bounded set of $C_{\omega}$ and $z \notin \partial \Omega$ for any $\lambda \in(0,1)$. Then the proof is complete.

Proposition 3.2. If the assumptions ( 1$)$-( $\times 3)$ hold, then the system (1.1) (or (1.2)) satisfies the condition (2) of Lemma 2.3.

Proof. We prove by the way of contradiction. Suppose that there exists a solution $\kappa=\left(\kappa_{1}, \ldots, \kappa_{n}, \kappa_{n+1}, \ldots\right.$, $\left.\kappa_{n+m}\right)^{\prime} \in \partial \Omega \cap \mathbb{R}^{n+m}$ of the inclusion $0 \in \frac{1}{\omega} \int_{0}^{\omega} F(t, k) d t=g_{0}(\kappa)$, then $k$ is a constant vector on $\mathbb{R}^{n+m}$ such that $\left|\kappa_{i}\right|=\vartheta_{i}^{*}$ for $i \in\{1,2, \ldots, n+m\}$, set $i=1$, then corresponding to $\kappa_{1}$ and $0 \in\left(g_{0}\left(\kappa_{1}\right)\right)=$ $\frac{1}{\omega} \int_{0}^{\omega} F_{1}\left(t, k_{1}\right) d t$, we have

$$
0=-\kappa_{1} \frac{1}{\omega} \int_{0}^{\omega} a_{1}(t) d t+\sum_{j=1}^{m} \gamma_{n+j} \frac{1}{\omega} \int_{0}^{\omega} p_{j 1}(t) d t+\sum_{j=1}^{m} \eta_{n+j} \frac{1}{\omega} \int_{0}^{\omega} q_{j 1}(t) d t+\frac{1}{\omega} \int_{0}^{\omega} c_{1}(t) d t .
$$

Then, there exists some $t^{*} \in[0, \omega]$ such that

$$
-\kappa_{1} a_{1}\left(t^{*}\right)+\sum_{j=1}^{m} p_{j 1}\left(t^{*}\right) \gamma_{n+j}+\sum_{j=1}^{m} q_{j 1}\left(t^{*}\right) \eta_{n+j}+c_{1}\left(t^{*}\right)=0 .
$$

From (3.15) we deduce that

$$
\begin{aligned}
\eta_{1}^{*}=\left|\kappa_{1}\right| & \leqslant \frac{1}{\left|a_{1}\left(t^{*}\right)\right|}\left[\sum_{j=1}^{m}\left|p_{j 1}\left(t^{*}\right)\right|\left|\gamma_{n+j}\right|+\sum_{j=1}^{m}\left|q_{j 1}\left(t^{*}\right)\right|\left|\eta_{n+j}\right|+\left|c_{1}\left(t^{*}\right)\right|\right] \\
& \leqslant \frac{1}{a_{1}^{L}}\left[\sum_{j=1}^{m} \alpha_{n+j}\left(p_{j 1}^{M}+\frac{q_{j 1}^{M}}{\sqrt{1-\tau^{D}}}\right)\left|\kappa_{n+j}\right|+\sum_{j=1}^{m} p_{j 1}^{M} \beta_{n+j}+\sum_{j=1}^{m} q_{j 1}^{M} \beta_{n+j}+c_{1}^{M}\right] \\
& =\sum_{j=1}^{m} m_{1(n+j)}\left|\kappa_{n+j}\right|+G_{1}^{\prime}=\sum_{j=1}^{n+m} m_{1 j}\left|\kappa_{j}\right|+G_{1},
\end{aligned}
$$

without loss of generality we can get the inequality for every $i \in\{2, \ldots, n+m\}$ which implies that $\left(E_{n+m}-\right.$ $M) \eta^{*} \leqslant G$, which contradicts to the fact $\left(E_{n+m}-M\right) \eta^{*}>G$. This proof is complete.

Next, we define a homotopic set-valued map $\phi: \Omega \cap \mathbb{R}^{\mathfrak{n}+\mathrm{m}} \times[0,1] \rightarrow \mathrm{C}_{\omega}$ by

$$
\phi(\kappa, \hbar)=\hbar \operatorname{diag}\left(-\overline{\mathrm{a}}_{1}, \ldots,-\overline{\mathrm{a}}_{\mathrm{n}},-\overline{\mathrm{b}}_{1}, \ldots,-\overline{\mathrm{b}}_{\mathrm{m}}\right) \mathrm{u}+(1-\hbar) \mathrm{g}_{0}(\kappa),
$$

where $\hbar \in[0,1]$ is a parameter, $\bar{a}_{i}=\frac{1}{\omega} \int_{0}^{\infty} a_{i}(t) d t, i=1,2, \ldots, n, \bar{b}_{j}=\frac{1}{\omega} \int_{0}^{\infty} b_{j}(t) d t, j=1,2, \ldots, m$. 
If $k=\left(\kappa_{1}, \ldots, \kappa_{n}, \kappa_{n+1}, \ldots, \kappa_{n+m}\right)^{\prime} \in \partial \Omega \cap \mathbb{R}^{n+m}$, then $\kappa$ is a constant vector on $\mathbb{R}^{n+m}$ such that $\left|\kappa_{i}\right|=\eta_{i}^{*}$ for some $i \in\{1,2, \ldots, n+m\}$. It follows that

$$
\begin{aligned}
(\phi(\kappa, \hbar))_{i}= & -\bar{a}_{i} \kappa_{i}+(1-\hbar)\left[\sum_{j=1}^{m} \overline{c o}\left[f_{j}\left(\kappa_{n+j}\right)\right] \frac{1}{\omega} \int_{0}^{\omega} p_{j i}(t) d t\right. \\
& \left.+\sum_{j=1}^{m} \overline{c o}\left[g_{j}\left(\kappa_{n+j}\right)\right] \frac{1}{\omega} \int_{0}^{\omega} q_{j i}(t) d t+\frac{1}{\omega} \int_{0}^{\omega} c_{i}(t) d t\right], i=1,2, \ldots, n, \\
(\phi(\kappa, \hbar))_{n+j}= & -\bar{b}_{j} \kappa_{j}+(1-\hbar)\left[\sum_{i=1}^{n} \overline{c o}\left[u_{i}\left(\kappa_{i}\right)\right] \frac{1}{\omega} \int_{0}^{\omega} r_{i j}(t) d t\right. \\
& \left.+\sum_{i=1}^{n} \overline{c o}\left[v_{i}\left(\kappa_{i}\right)\right] \frac{1}{\omega} \int_{0}^{\omega} s_{i j}(t) d t+\frac{1}{\omega} \int_{0}^{\omega} d_{j}(t) d t\right], j=1,2, \ldots, m .
\end{aligned}
$$

Remark 3.3. Based on the proof of Proposition 3.2, by the method of contradiction, without loss of generality, we also claim that

$$
0 \notin(\phi(\kappa, \hbar))_{i}, \quad i=1,2, \ldots, n+m .
$$

Proposition 3.4. Under Proposition 3.2 and Remark 3.3, the system (1.1) (or (1.2)) satisfies the condition (3) of Lemma 2.3.

Proof. If (3.16) holds, if follows that

$$
(0, \ldots, 0,0, \ldots, 0)^{\prime} \notin \phi(\kappa, \hbar), \forall \kappa=\left(\kappa_{1}, \ldots, \kappa_{n}, \kappa_{n+1}, \ldots, \kappa_{n+m}\right)^{\prime} \in \partial \Omega \cap \mathbb{R}^{n+m} .
$$

Therefore, by the homotopy invariance and the solution properties of the topological degree, we obtain

$$
\begin{aligned}
\operatorname{deg}\left\{g_{0}, \Omega \cap \mathbb{R}^{n+m}, 0\right\} \\
\quad=\operatorname{deg}\left\{\phi(\kappa, 0), \Omega \cap \mathbb{R}^{n+m}, 0\right\} \\
=\operatorname{deg}\left\{\phi(\kappa, 1), \Omega \cap \mathbb{R}^{n+m}, 0\right\} \\
=\operatorname{deg}\left\{\left(-\bar{a}_{1} \kappa_{1}, \ldots,-\bar{a}_{n} \kappa_{n},-\bar{b}_{1} \kappa_{n+1}, \ldots,-\bar{b}_{m} \kappa_{n+m}\right)^{\prime}, \Omega \cap \mathbb{R}^{n+m},(0, \ldots, 0)^{\prime}\right\} \\
\quad=\operatorname{sign}\left|\begin{array}{ccc}
-\bar{a}_{1} & \cdots & 0 \\
\vdots & \ddots & \vdots \\
0 & \cdots & -\bar{b}_{m}
\end{array}\right|=(-1)^{n+m} \neq 0,
\end{aligned}
$$

where $\operatorname{deg}(\cdot, \cdot, \cdot)$ denotes the topological degree for upper semi-continuous set-valued maps with compact convex values (see [24]). Then the proof is complete.

Then, we have proved that $\Omega$ satisfies all the conditions in Lemma 2.3, we can give the following theorem on the existence of periodic solution of complex neural network model (1.1) (or (1.2)).

Theorem 3.5. Suppose that the assumptions ( 1$)-(\aleph 3)$ are satisfied, then the discontinuous network system (1.1) (or (1.2)) has at least one w-periodic solution.

Remark 3.6. In general, it is easily verified that neuron dynamic approaches are based on M-matrix, and there are many existing results (see $[5,9]$ ) to analyze the periodic solutions of neuron dynamic systems with discontinuous neuron activations via Yoshizawa-like theorem, Krasnoselskii's Fixed point theorem of set-valued maps, Leray-Schauder alternative theorem, etc., see [6]. However, it is necessary to introduce the existence of the periodic solution which has been ignored by many researches, in this section, we obtained that the results on the existence of the periodic solutions for the complex BAM network dynamical system with time-varying delays and discontinuous activations which is prepared for the next section. 


\section{Periodic synchronization under control}

In this section, by designing a state-feedback controller, we study the global exponential synchronization of complex BAM system with periodic coefficients and discontinuous activations. Suppose that $z(t)=\left(x_{1}(t), \ldots, x_{n}(t), y_{1}(t), \ldots, y_{m}(t)\right)^{\prime}$ is an arbitrary solution of Eq. (1.1) with initial condition $\phi(s)=$ $\left(\varphi_{1}, \ldots, \varphi_{n}, \varphi_{1}, \ldots, \psi_{m}\right)^{\prime}$. By Theorem 3.5, there exists an $\omega$-periodic solution $z^{*}(t)=\left(x_{1}^{*}(t), \ldots, x_{\mathfrak{n}}^{*}(t)\right.$, $\left.y_{1}^{*}(t), \ldots, y_{m}^{*}(t)\right)^{\prime}$ with initial condition $\phi^{*}(s)=\left(\varphi_{1}^{*}, \ldots, \varphi_{n}^{*}, \varphi_{1}^{*}, \ldots, \psi_{m}^{*}\right)^{\prime}$ for Eq. (1.1). For $i=1,2, \ldots, n$, $j=1,2, \ldots, m$, now we consider the complex network model (1.1) as the driver system, the corresponding response system can be described as the following functional differential equations:

$$
\left\{\begin{array}{l}
\frac{d \sigma_{i}(t)}{d t}=-a_{i}(t) \sigma_{i}(t)+\sum_{j=1}^{m} p_{j i}(t) f_{j}\left(\pi_{j}(t)\right)+\sum_{j=1}^{m} q_{j i}(t) g_{j}\left(\pi_{j}(t-\tau(t))\right)+\mu_{i}(t), \\
\frac{d \pi_{j}(t)}{d t}=-b_{j}(t) \pi_{j}(t)+\sum_{i=1}^{n} r_{i j}(t) u_{i}\left(\sigma_{i}(t)\right)+\sum_{i=1}^{n} s_{i j}(t) v_{i}\left(\sigma_{i}(t-\tau(t))\right)+v_{j}(t),
\end{array}\right.
$$

where $\mu_{i}(t), v_{j}(t)$ are the controller to be designed for reaching periodic synchronization of the driveresponse system. The other parameters are the same as those defined in model (1.1).

According to the theories of differential inclusions and set-valued maps, for $i=1,2, \ldots, n, j=$ $1,2, \ldots, m$, we can give the initial value problem (IVP) of response system (4.1) for a.e. $t \in[0,+\infty)$ as follows:

$$
\left\{\begin{array}{l}
\frac{d \sigma_{i}(t)}{d t}=-a_{i}(t) \sigma_{i}(t)+\sum_{j=1}^{m} p_{j i}(t) \widetilde{\gamma}_{n+j}(t)+\sum_{j=1}^{m} q_{j i}(t) \widetilde{\eta}_{n+j}(t-\tau(t))+\mu_{i}(t), \\
\frac{d \pi_{j}(t)}{d t}=-b_{j}(t) \pi_{j}(t)+\sum_{i=1}^{n} r_{i j}(t) \widetilde{\gamma}_{i}(t)+\sum_{i=1}^{n} s_{i j}(t) \widetilde{\eta}_{i}(t-\tau(t))+v_{j}(t), \\
\widetilde{\gamma}_{n+j} \in \overline{c o}\left[f_{j}\left(\pi_{j}(t)\right)\right], \widetilde{\gamma}_{i} \in \overline{c o}\left[u_{i}\left(\sigma_{i}(t)\right)\right], \widetilde{\eta}_{n+j} \in \overline{c o}\left[g_{j}\left(\pi_{j}(t)\right)\right], \widetilde{\eta}_{i} \in \overline{c o}\left[v_{i}\left(\sigma_{i}(t)\right)\right], \\
\sigma(s)=\left(\sigma_{1}(s), \sigma_{2}(s), \ldots, \sigma_{n}(s)\right)^{\prime}, \forall s \in[-\tau, 0], \\
\pi(s)=\left(\pi_{1}(s), \pi_{2}(s), \ldots, \pi_{m}(s)\right)^{\prime}, \forall s \in[-\tau, 0] .
\end{array}\right.
$$

Next we define the convergence rate of exponential synchronization error between the drive system and the response system, for $i=1,2, \ldots, n, j=1,2, \ldots, m$, we obtain

$$
e_{i}(t)=\sigma_{i}(t)-x_{i}(t) \text { and } e_{n+j}(t)=\pi_{j}(t)-y_{j}(t),
$$

then we can get the following synchronization error system

$$
\left\{\begin{array}{l}
\frac{d e_{i}(t)}{d t}=-a_{i}(t) e_{i}(t)+\sum_{j=1}^{m} p_{j i}(t) \Gamma_{n+j}(t)+\sum_{j=1}^{m} q_{j i}(t) \Upsilon_{n+j}(t-\tau(t))+\mu_{i}(t), \\
\frac{d e_{n+j}(t)}{d t}=-b_{j}(t) e_{n+j}(t)+\sum_{i=1}^{n} r_{i j}(t) \Gamma_{i}(t)+\sum_{i=1}^{n} s_{i j}(t) \Upsilon_{i}(t-\tau(t))+v_{j}(t),
\end{array}\right.
$$

where $\Gamma_{k}(t)=\widetilde{\gamma}_{k}(t)-\gamma_{k}(t)$ and $\Gamma_{k}(t-\tau)=\widetilde{\eta}_{k}(t-\tau)-\eta_{k}(t-\tau), k=1,2, \ldots, n+m$. We consider the following discontinuous feedback controllers:

$$
\mu_{i}(t)=-l_{i} e_{i}(t)-m_{i} \operatorname{sign}\left(e_{i}(t)\right) \text { and } v_{j}(t)=-l_{n+j} e_{n+j}(t)-m_{n+j} \operatorname{sign}\left(e_{n+j}(t)\right),
$$

where $i=1,2, \ldots, n, j=1,2, \ldots, m$.

By the assumption ( $\ 2$, we assumed further that the discontinuous neuron activations $h$ satisfies the following condition: 
( $\aleph$ ) For every $i \in \mathbb{N}$, there exist nonnegative constants $\alpha_{i}$ and $\beta_{i}$ such that

$$
\sup _{\xi_{i} \in \overline{c o}\left[h_{i}(\iota)\right], \zeta_{i} \in \overline{c o}\left[h_{i}(\kappa)\right]}\left|\xi_{i}-\zeta_{i}\right| \leqslant \alpha_{i}|\iota-k|+\beta_{i}, \forall \iota, k \in \mathbb{R}
$$

and

$$
\sup _{\left.\widetilde{\xi}_{i} \in \overline{\operatorname{co}}_{h_{\mathfrak{h}}}(\iota)\right], \widetilde{\zeta}_{i} \in \overline{\operatorname{co}}\left[\widetilde{h}_{i}(\kappa)\right]}\left|\widetilde{\xi}_{i}-\widetilde{\zeta}_{i}\right| \leqslant \alpha_{i}|\iota-\kappa|+\beta_{i}, \quad \forall \iota, k \in \mathbb{R} .
$$

Remark 4.1. Because of the functions $h_{i}$ and $\widetilde{h}_{i}$ are discontinuous, the constant $\beta_{i}$ in the assumption ( $\$ ) should not be equal to zero. If not, the assumption ( $\$$ ) is completely different from the Lipschitz condition in the previous literature which is in contradiction with our hypothesis.

For convenience, we denote

$$
\|\phi-\psi\|_{C}=\sup _{-\tau \leqslant \theta \leqslant 0} \sum_{i=1}^{n+m}\left|\phi_{i}(\theta)-\psi_{i}(\theta)\right| .
$$

Definition $4.2([25,28])$. The drive system (1.1) and the response system (4.1) with discontinuous activations function are said to be globally exponentially synchronization if there exist positive constants $\Re \geqslant 1$ and $\lambda>0$ such that

$$
\sum_{i=1}^{n}\left|\sigma_{i}(t)-x_{i}(t)\right|+\sum_{j=1}^{m}\left|\pi_{j}(t)-y_{j}(t)\right|=\Re\|\phi-\psi\|_{C} \exp \{-\lambda t\},
$$

where $\lambda$ is called the convergence rate of exponential synchronization.

Theorem 4.3. Suppose that conditions ( 1$)-(\aleph 4)$ are satisfied, assume further that

( $\times 5)$ For every $i=1,2, \ldots, n, j=1,2, \ldots, m$, we assume

$$
m_{i} \geqslant \sum_{j=1}^{m} p_{j i}^{M} \beta_{n+j}+\sum_{j=1}^{m} q_{j i}^{M} \beta_{n+j}, \quad m_{n+j} \geqslant \sum_{i=1}^{n} r_{i j}^{M} \beta_{i}+\sum_{i=1}^{n} s_{i j}^{M} \beta_{i}
$$

and

$$
\Delta^{\mathrm{L}}>\exp \left\{\Delta^{\mathrm{M}} \tau\right\} \Lambda
$$

where

$$
\begin{aligned}
& \Lambda_{1}=\max \left\{\max _{1 \leqslant j \leqslant m}\left\{\sum_{i=1}^{n} r_{i j}^{M} \alpha_{i}\right\}, \max _{1 \leqslant i \leqslant n}\left\{\sum_{j=1}^{m} p_{j i}^{M} \alpha_{n+j}\right\}\right\}, \Lambda_{2}=\max \left\{\max _{1 \leqslant j \leqslant m}\left\{\sum_{i=1}^{n} s_{i j}^{M} \alpha_{i}\right\}, \max _{1 \leqslant i \leqslant n}\{\right. \\
& \left.\left.\sum_{j=1}^{m} q_{j i}^{M} \alpha_{n+j}\right\}\right\}, \Lambda=\max \left\{\Lambda_{1}, \Lambda_{2}\right\}, \Delta(t)=\min \left\{\min _{1 \leqslant i \leqslant n}\left\{\left(a_{i}(t)+l_{i}\right)\right\}, \min _{1 \leqslant j \leqslant m}\left\{\left(b_{j}(t)+l_{n+j}\right)\right\}\right\} .
\end{aligned}
$$

Then under the discontinuous switching feedback controller (4.4), the neural networks (1.1) and (4.2) can achieve global exponential synchronization. Moreover, the convergence rate is $\lambda=\Delta^{\mathrm{L}}-\exp \left\{\Delta^{\mathrm{M}} \tau\right\} \wedge>0$.

Proof. Substituting the discontinuous switching feedback controller (4.4) into the synchronization error system (4.3), for a.e. $t \geqslant 0$, we obtain

$$
\left\{\begin{array}{l}
\frac{d e_{i}(t)}{d t}=-a_{i}(t) e_{i}(t)+\sum_{j=1}^{m} p_{j i}(t) \Gamma_{n+j}(t)+\sum_{j=1}^{m} q_{j i}(t) r_{n+j}(t-\tau(t))-l_{i} e_{i}(t)-m_{i}\left(e_{i}(t)\right), \\
\frac{d e_{n+j}(t)}{d t}=-b_{j}(t) e_{n+j}(t)+\sum_{i=1}^{n} r_{i j}(t) \Gamma_{i}(t)+\sum_{i=1}^{n} s_{i j}(t) r_{i}(t-\tau(t))-l_{n+j} e_{n+j}(t)-m_{n+j}\left(e_{n+j}(t)\right) .
\end{array}\right.
$$


For any $t \geqslant 0$, we consider the following positive radially unbounded candidate Lypunov function:

$$
V(t)=V_{1}(t)+V_{2}(t)=\sum_{i=1}^{n}\left|e_{i}(t)\right|+\sum_{j=1}^{m}\left|e_{n+j}(t)\right| .
$$

Now we define $V_{t}(\theta)=V(t+\theta),-\tau \leqslant \theta \leqslant 0$ and $t \in[0, T)$. Moreover, for any $t \in[0, T)$, the supnorm is given as $\left\|V_{t}\right\|_{C}=\sup _{-\tau \leqslant \theta \leqslant 0}\left|V_{t}(\theta)\right|$. It is easy to see that the function $V(t)=V_{t}(0)$ is absolutely continuous and C-regular; $V(t)>0, V(0)$ is positive and finite. We denote $w_{i}(t)=\operatorname{sign}\left(\sigma_{i}(t)\right)$, if $\sigma_{i}(t) \neq 0$; while $w_{i}(t)$ can be arbitrarily chosen in $[-1,1]$, if $\sigma_{i}(t)=0$. Therefore, taking (4.5) into account, under the conditions of the theorem, calculating the derivative of $V(t)$ for a.e. $t \geqslant 0$, we can obtain

$$
\begin{aligned}
& \frac{d V_{1}(t)}{d t}=\sum_{i=1}^{n} \frac{d e_{i}(t)}{d t} w_{i}(t) \\
& =\sum_{i=1}^{n}\left[-\left(a_{i}(t)+l_{i}\right) e_{i}(t)+\sum_{j=1}^{m} p_{j i}(t) \Gamma_{n+j}(t)\right. \\
& \left.+\sum_{j=1}^{m} q_{j i}(t) \Upsilon_{n+j}(t-\tau(t))-m_{i} \operatorname{sign}\left(e_{i}(t)\right)\right] \operatorname{sign}\left(e_{i}(t)\right) \\
& =\sum_{i=1}^{n}-\left(a_{i}(t)+l_{i}\right)\left|e_{i}(t)\right|+\sum_{i=1}^{n} \sum_{j=1}^{m} p_{j i}(t) \Gamma_{n+j}(t) \operatorname{sign}\left(e_{i}(t)\right) \\
& +\sum_{i=1}^{n} \sum_{j=1}^{m} q_{j i}(t) \Upsilon_{n+j}(t-\tau(t)) \operatorname{sign}\left(e_{i}(t)\right)-\sum_{i=1}^{n} m_{i}\left|\operatorname{sign}\left(e_{i}(t)\right)\right| \\
& \leqslant \sum_{i=1}^{n}-\left(a_{i}(t)+l_{i}\right)\left|e_{i}(t)\right|+\sum_{i=1}^{n} \sum_{j=1}^{m}\left|p_{j i}(t)\right|\left|\Gamma_{n+j}(t) \| \operatorname{sign}\left(e_{i}(t)\right)\right| \\
& +\sum_{i=1}^{n} \sum_{j=1}^{m}\left|q_{j i}(t)\right|\left|r_{n+j}(t-\tau(t))\right|\left|\operatorname{sign}\left(e_{i}(t)\right)\right|-\sum_{i=1}^{n} m_{i}\left|\operatorname{sign}\left(e_{i}(t)\right)\right| \\
& \leqslant \sum_{i=1}^{n}-\left(a_{i}(t)+l_{i}\right)\left|e_{i}(t)\right|+\sum_{i=1}^{n} \sum_{j=1}^{m} p_{j i}^{M}\left(\alpha_{n+j}\left|e_{n+j}(t)\right|+\beta_{n+j}\right)\left|\operatorname{sign}\left(e_{i}(t)\right)\right| \\
& +\sum_{i=1}^{n} \sum_{j=1}^{m} q_{j i}^{M}\left(\alpha_{n+j}\left|e_{n+j}(t-\tau(t))\right|+\beta_{n+j}\right)\left|\operatorname{sign}\left(e_{i}(t)\right)\right|-\sum_{i=1}^{n} m_{i}\left|\operatorname{sign}\left(e_{i}(t)\right)\right| \\
& \leqslant \sum_{i=1}^{n}-\left(a_{i}(t)+l_{i}\right)\left|e_{i}(t)\right|+\sum_{i=1}^{n} \sum_{j=1}^{m} p_{j i}^{M} \alpha_{n+j}\left|e_{n+j}(t)\right|+\sum_{i=1}^{n} \sum_{j=1}^{m} q_{j i}^{M} \alpha_{n+j}\left|e_{n+j}(t-\tau(t))\right| \\
& -\sum_{i=1}^{n}\left(m_{i}-\sum_{j=1}^{m} p_{j i}^{M} \beta_{n+j}-\sum_{j=1}^{m} q_{j i}^{M} \beta_{n+j}\right)\left|\operatorname{sign}\left(e_{i}(t)\right)\right| \\
& \leqslant \sum_{i=1}^{n}-\left(a_{i}(t)+l_{i}\right)\left|e_{i}(t)\right|+\sum_{i=1}^{n} \sum_{j=1}^{m} p_{j i}^{M} \alpha_{n+j}\left|e_{n+j}(t)\right|+\sum_{i=1}^{n} \sum_{j=1}^{m} q_{j i}^{M} \alpha_{n+j}\left|e_{n+j}(t-\tau(t))\right| .
\end{aligned}
$$

Similar to (4.6), we obtain

$$
\begin{aligned}
\frac{d V_{2}(t)}{d t} \leqslant & \sum_{j=1}^{m}-\left(b_{j}(t)+l_{n+j}\right)\left|e_{n+j}(t)\right|+\sum_{j=1}^{m} \sum_{i=1}^{n} r_{i j}^{M} \alpha_{i}\left|e_{i}(t)\right|+\sum_{j=1}^{m} \sum_{i=1}^{n} s_{i j}^{M} \alpha_{i}\left|e_{i}(t-\tau(t))\right| \\
& -\sum_{j=1}^{m}\left(m_{n+j}-\sum_{i=1}^{n} r_{i j}^{M} \beta_{i}-\sum_{i=1}^{n} s_{i j}^{M} \beta_{i}\right)\left|\operatorname{sign}\left(e_{n+j}(t)\right)\right| . \\
\leqslant & \sum_{j=1}^{m}-\left(b_{j}(t)+l_{n+j}\right)\left|e_{n+j}(t)\right|+\sum_{j=1}^{m} \sum_{i=1}^{n} r_{i j}^{M} \alpha_{i}\left|e_{i}(t)\right|+\sum_{j=1}^{m} \sum_{i=1}^{n} s_{i j}^{M} \alpha_{i}\left|e_{i}(t-\tau(t))\right| .
\end{aligned}
$$


Moreover, combining (4.6) and (4.7), under assumption of the theorem, we obtain

$$
\begin{aligned}
\frac{d V(t)}{d t}=\frac{d V_{1}(t)}{d t}+\frac{d V_{2}(t)}{d t} \leqslant & -\min \left\{\min _{1 \leqslant i \leqslant n}\left\{\left(a_{i}(t)+l_{i}\right)\right\}, \min _{1 \leqslant j \leqslant m}\left\{\left(b_{j}(t)+l_{n+j}\right)\right\}\right\} \sum_{k=1}^{n+m} e_{k}(t) \\
& +\max \left\{\max _{1 \leqslant j \leqslant m}\left\{\sum_{i=1}^{n} r_{i j}^{M} \alpha_{i}\right\}, \max _{1 \leqslant i \leqslant n}\left\{\sum_{j=1}^{m} p_{j i}^{M} \alpha_{n+j}\right\}\right\} \sum_{k=1}^{n+m} e_{k}(t) \\
& +\max \left\{\max _{1 \leqslant j \leqslant m}\left\{\sum_{i=1}^{n} s_{i j}^{M} \alpha_{i}\right\}, \max _{1 \leqslant i \leqslant n}\left\{\sum_{j=1}^{m} q_{j i}^{M} \alpha_{n+j}\right\}\right\} \sum_{k=1}^{n+m} e_{k}(t-\tau(t)) \\
= & -\Delta(t) V(t)+\Lambda\left\|V_{t}\right\|_{C}, \text { for a.e. } t \geqslant 0,
\end{aligned}
$$

where $\Delta(t)=\min \left\{\min _{1 \leqslant i \leqslant n}\left\{\left(a_{i}(t)+l_{i}\right)\right\}, \min _{1 \leqslant j \leqslant m}\left\{\left(b_{j}(t)+l_{n+j}\right)\right\}\right\}$ and $\Lambda_{1}=\max \left\{\max _{1 \leqslant j \leqslant m}\left\{\sum_{i=1}^{n} r_{i j}^{M} \alpha_{i}\right\}, \max _{1 \leqslant i \leqslant n}\{\right.$ $\left.\left.\sum_{j=1}^{m} p_{j i}^{M} \alpha_{n+j}\right\}\right\}, \Lambda_{2}=\max \left\{\max _{1 \leqslant j \leqslant m}\left\{\sum_{i=1}^{n} s_{i j}^{M} \alpha_{i}\right\}, \max _{1 \leqslant i \leqslant n}\left\{\sum_{j=1}^{m} q_{j i}^{M} \alpha_{n+j}\right\}\right\}, \Lambda=\max \left\{\Lambda_{1}, \Lambda_{2}\right\}$.

Multiplying both sides of the inequality (4.8) by $\exp \left\{\int_{0}^{t} \Delta(\rho) \mathrm{d} \rho\right\}$ and integrating both sides of the inequality over the interval $[0, t]$, it follows that

$$
\exp \left\{\int_{0}^{t} \Delta(\rho) \mathrm{d} \rho\right\} V(t) \leqslant V(0)+\int_{0}^{t} \exp \left\{\int_{0}^{s} \Delta(\rho) \mathrm{d} \rho\right\} \wedge\left\|V_{s}\right\|_{C} \mathrm{~d} s,
$$

therefore, for $-\tau \leqslant \theta \leqslant 0$, we can deduce that

$$
\begin{aligned}
\exp \left\{\int_{0}^{t} \Delta(\rho) \mathrm{d} \rho-\Delta^{M} \tau\right\} V(t+\theta) & \leqslant \exp \left\{\int_{0}^{t+\theta} \Delta(\rho) \mathrm{d} \rho\right\} V(t+\theta) \\
& \leqslant V(0)+\int_{0}^{t+\theta} \exp \left\{\int_{0}^{s} \Delta(\rho) \mathrm{d} \rho\right\} \wedge\left\|V_{s}\right\|_{C} d s \\
& \leqslant\left\|V_{0}\right\|_{C}+\int_{0}^{t} \exp \left\{\int_{0}^{s} \Delta(\rho) \mathrm{d} \rho\right\} \wedge\left\|V_{s}\right\|_{C} d s
\end{aligned}
$$

which implies

$$
\exp \left\{\int_{0}^{t} \Delta(\rho) \mathrm{d} \rho\right\}\left\|\mathrm{V}_{\mathrm{t}}\right\|_{\mathrm{C}} \leqslant \exp \left\{\Delta^{\mathrm{M}} \tau\right\}\left\|\mathrm{V}_{0}\right\|_{\mathrm{C}}+\int_{0}^{\mathrm{t}} \exp \left\{\Delta^{\mathrm{M}} \tau\right\} \exp \left\{\int_{0}^{s} \Delta(\rho) \mathrm{d} \rho\right\} \wedge\left\|\mathrm{V}_{\mathrm{s}}\right\|_{\mathrm{C}} \mathrm{ds} .
$$

By Gronwall inequality, we have

$$
\exp \left\{\int_{0}^{t} \Delta(\rho) \mathrm{d} \rho\right\}\left\|\mathrm{V}_{\mathrm{t}}\right\|_{\mathrm{C}} \leqslant \exp \left\{\Delta^{M} \tau\right\}\left\|\mathrm{V}_{0}\right\|_{\mathrm{C}} \cdot \exp \left\{\exp \left\{\Delta^{M} \tau\right\} \wedge t\right\},
$$

which yields

$$
\begin{aligned}
\left\|V_{t}\right\|_{C} & \leqslant \exp \left\{\Delta^{M} \tau\right\}\left\|V_{0}\right\|_{C} \cdot \exp \left\{\exp \left\{\Delta^{M} \tau\right\} \wedge t\right\} \cdot \exp \left\{-\int_{0}^{t} \Delta(\rho) \operatorname{d} \rho\right\} \\
& \leqslant \exp \left\{\Delta^{M} \tau\right\}\left\|V_{0}\right\|_{C} \cdot \exp \left\{-\left(\Delta^{L}-\exp \left\{\Delta^{M} \tau\right\} \wedge\right) t\right\},
\end{aligned}
$$

this means that

$$
\begin{aligned}
& \sum_{i=1}^{n}\left|\sigma_{i}(t)-x_{i}(t)\right|+\sum_{j=1}^{m}\left|\pi_{j}(t)-y_{j}(t)\right| \\
& \quad=V(t) \leqslant\left\|V_{t}\right\|_{C} \leqslant \exp \left\{\Delta^{M} \tau\right\}\left\|V_{0}\right\|_{C} \cdot \exp \left\{-\left(\Delta^{L}-\exp \left\{\Delta^{M} \tau\right\} \Lambda\right) t\right\} \\
& \quad=\Re\|\phi-\psi\|_{C} \exp \{-\lambda t\},
\end{aligned}
$$


where $\mathfrak{R}=\exp \left\{\Delta^{\mathrm{M}} \tau\right\},\|\phi-\psi\|_{\mathrm{C}}=\left\|\mathrm{V}_{0}\right\|_{\mathrm{C}}=\sup _{-\tau \leqslant \theta \leqslant 0} \sum_{i=1}^{\mathrm{n}+\mathrm{m}}\left|\varphi_{i}(\theta)-\psi_{i}(\theta)\right|$ and $\lambda=\Delta^{\mathrm{L}}-\exp \left\{\Delta^{\mathrm{M}} \tau\right\} \wedge$ are positive constants. Under the discontinuous switching feedback controller (4.4), according to Definition 4.2, the driver network system (1.1) can realize globally exponentially synchronization with the response system (4.1). The proof is complete.

Remark 4.4. In this paper, we designed a discontinuous switching terms $\mu_{\mathfrak{i}}(t)$ and $v_{j}(t)$, taking measurable selection in the appropriate closure of the convex hull, by Filippov regularization and in the sense of initial value conditions, the system (1.1) is first transformed into (4.1). Between response network systems and the Filippov solutions of the drive system, such a discontinuous switching feedback controller can deal with the uncertain differences. From proof of Theorem 4.3, in realizing the periodic synchronization goal, one can see that the discontinuous switching term in state-feedback controller (4.1) plays a very important role. However, it is not easy to construct the conventional Lyapunov-like functions for realizing synchronization control of complex BAM neural networks, and it is also not easy to design suitable controller to achieve the periodic synchronization for complex BAM networks with time-varying delays and discontinuous activations. Our future researches should be emphasized on designing better and simpler controlled response system.

\section{Examples and simulation experiment}

In this section, using MATLAB programming, we provide a simulation example to illustrate our criteria. Consider the following three-dimensional discontinuous time-delayed network system as follows:

$$
\left\{\begin{aligned}
\frac{d x(t)}{d t}= & -a(t) x(t)+p_{11}(t) f_{1}\left(y_{1}(t)\right)+p_{21}(t) f_{2}\left(y_{2}(t)\right) \\
& +q_{11}(t) g_{1}\left(y_{1}(t-\tau(t))\right)+q_{21}(t) g_{2}\left(y_{2}(t-\tau(t))\right)+c(t), \\
\frac{d y_{1}(t)}{d t}= & -b_{1}(t) y_{1}(t)+r_{1}(t) u(x(t))+s_{1}(t) v(x(t-\tau(t)))+d_{1}(t), \\
\frac{d y_{2}(t)}{d t}= & -b_{2}(t) y_{2}(t)+r_{2}(t) u(x(t))+s_{2}(t) v(x(t-\tau(t)))+d_{2}(t),
\end{aligned}\right.
$$

where $a(t)=b_{1}(t)=b_{2}(t)=1, c(t)=4, d_{1}(t)=4+\sin t, d_{2}(t)=4+$ cost, $p_{11}(t)=-\frac{1}{2}+$ sint, $p_{21}(t)=-\frac{1}{2}+\frac{1}{2} \cos t, q_{11}(t)=-\frac{1}{3} \sin t, q_{21}(t)=\frac{1}{6} \cos t, r_{1}(t)=\frac{1}{8}+\frac{1}{8} \sin t, r_{2}(t)=-\frac{1}{4}+\frac{1}{4} \cos t, s_{1}(t)=\frac{1}{2} \sin t$, $s_{2}(t)=-\frac{1}{4} \cos t$ and $\tau(t)=\frac{1}{2}$. The discontinuous activation functions are taken as

$$
\begin{gathered}
f_{1}(y)=f_{2}(y)=\left\{\begin{array}{c}
y-\frac{1}{2}, y \geqslant 0, \\
y+\frac{1}{2}, y<0,
\end{array} \quad u(x)=\left\{\begin{array}{c}
\frac{1}{10} x+\frac{1}{2}, x \geqslant 0, \\
\frac{1}{10} x-\frac{1}{2}, x<0 .
\end{array}\right.\right. \\
g_{1}(y)=g_{2}(y)=\left\{\begin{array}{c}
\tanh (y)-\frac{1}{10}, y \geqslant 0, \\
\tanh (y)+\frac{1}{10}, y<0 .
\end{array} \quad v(x)=\left\{\begin{array}{c}
x+\frac{1}{10}, x \geqslant 0, \\
x-\frac{1}{10}, x<0 .
\end{array}\right.\right.
\end{gathered}
$$

We can easily calculate that

$$
E-M=\left(\begin{array}{ccc}
1 & -\frac{5}{6} & -\frac{1}{6} \\
-\frac{3}{4} & 1 & 0 \\
-\frac{1}{4} & 0 & 1
\end{array}\right) .
$$

We can get that $E-M$ is an M-matrix. Therefore, it follows from Theorem 3.5 that the non-autonomous system (5.1) with neuron input $\mathrm{I}(\mathrm{t})=(4,4+\sin t, 4+\cos t)$ has at least one $2 \pi$-periodic solution.

Obviously, the discontinuous functions are non-monotonic and satisfy the assumption ( 11$)$. Meanwhile, 0 is a discontinuous point of the activation functions, we choose $\alpha_{1}=\alpha_{2}=\alpha_{3}=\beta_{1}=\beta_{2}=\beta_{3}=1$ 
such that the condition ( $(2)$ holds. Take $l_{1}=l_{2}=l_{3}=0.1, m_{1}=m_{2}=m_{3}=1.2$. Then we have $m_{1}>\sum_{j=1}^{m} p_{j i}^{M} \beta_{n+j}+\sum_{j=1}^{m} q_{j i}^{M} \beta_{n+j}=1, m_{2}=m_{3}>\sum_{i=1}^{n} r_{i j}^{M} \beta_{i}+\sum_{i=1}^{n} s_{i j}^{M} \beta_{i}=1, \Lambda_{1}=\Lambda_{2}=\frac{1}{2}$, and $\Lambda=\frac{1}{2}$, $\Delta(t)=1.1>\frac{1}{2} \exp 0.505=0.754$. Therefore, by Theorem 4.3 , the system (4.6) can also realize the global exponential synchronization with the corresponding response system under the discontinuous feedback controller which can be depicted in Figure 1. It means that the numerical simulation is suitable for our main results.

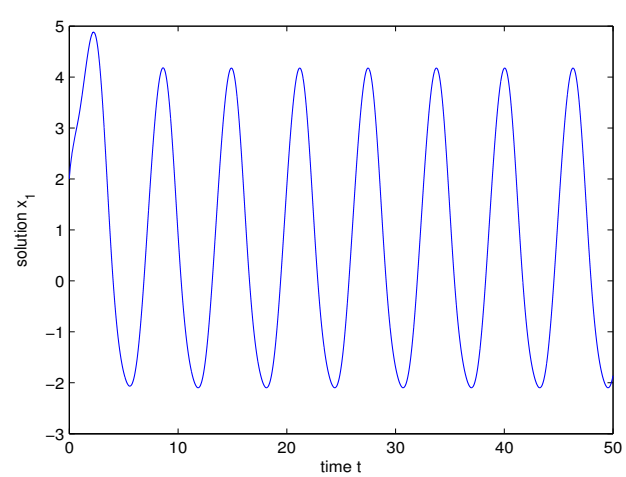

(a)

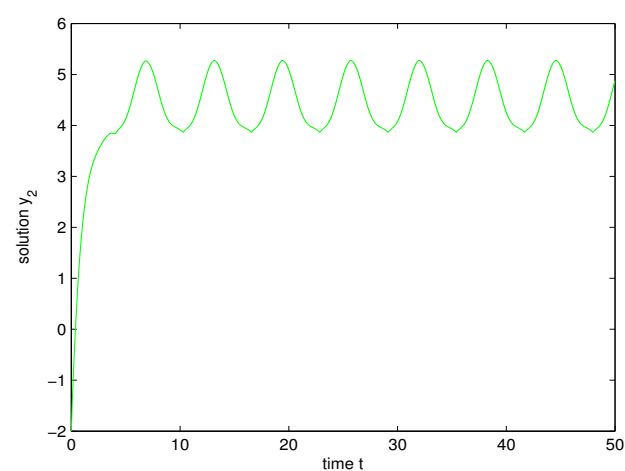

(c)

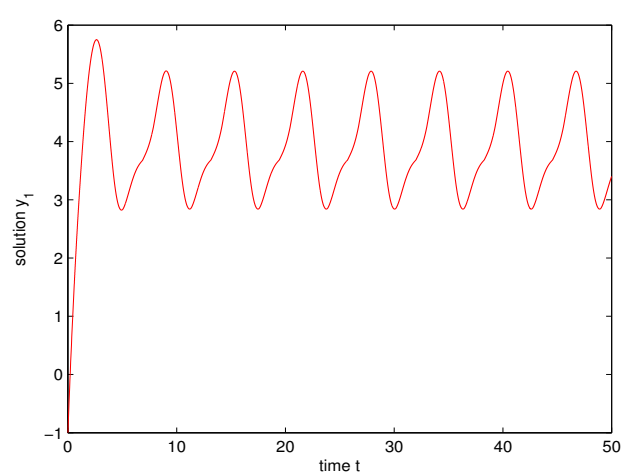

(b)

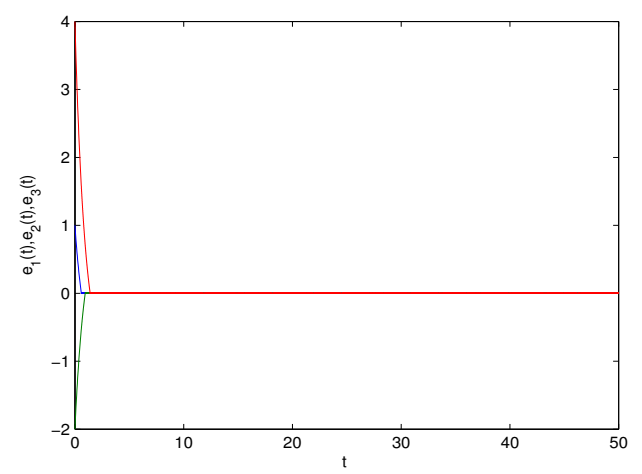

(d)

Figure 1: (a)-(c) are periodic solutions of system (1.1), (d) is time respond of synchronization error between drive system (4.3) and corresponding response system under the discontinuous switching state-feedback controller (4.4).

\section{Conclusions}

In this paper, we consider a class of complex BAM network dynamical systems with time-varying delays and discontinuous activations. Our main technologies of this paper involve periodic synchronization theorems and the theory of differential inclusions developed by Filippov, we have introduced a new research method to guarantee the existence of the periodic solution, that is, by using the set-valued analysis and degree theory, we have obtained that there exists at least one periodic solution for the complex BAM network dynamical systems with time-varying delays and discontinuous activations. Then, by constructing C-regular Lyapunov-like function and designing novel discontinuous switching feedback controller, which can realize the global exponential synchronization of the drive-response system for discontinuous time-delayed BAM neural networks with periodic coefficients. These results obtained in this paper improve and extend the previous works, and in the further work to solve the other synchronization issues, we wish that the methods and tools constructed in this paper can be widely used. 


\section{Acknowledgment}

This work is supported by the National Natural Science Foundation of China (11771059).

\section{References}

[1] J. P. Aubin, A. Cellina, Differential inclusions, Set-valued maps and viability theory, Grundlehren der Mathematischen Wissenschaften [Fundamental Principles of Mathematical Sciences], Springer-Verlag, Berlin, (1984). 2

[2] A. Berman, R. J. Plemmons, Nonnegative matrices in the mathematical sciences, Computer Science and Applied Mathematics, Academic Press [Harcourt Brace Jovanovich, Publishers], New York-London, (1979). 2.5

[3] V. I. Blagodatskikh, A. F. Filippov, Differential inclusions and optimal control, (Russian) Topology, ordinary differential equations, dynamical systems, Trudy Mat. Inst. Steklov., 169 (1985), 194-252. 2

[4] Z.-W. Cai, L.-H. Huang, Functional differential inclusions and dynamic behaviors for memristor-based BAM neural networks with time-varying delays, Commun. Nonlinear Sci. Numer. Simul., 19 (2014), 1279-1300. 1

[5] Z.-W. Cai, L.-H. Huang, Z.-Y. Guo, X.-Y. Chen, On the periodic dynamics of a class of time-varying delayed neural networks via differential inclusions, Neural Netw., 33 (2012), 97-113. 1, 3.6

[6] Z.-W. Cai, L.-H. Huang, Z.-Y. Guo, L.-L. Zhang, X.-T. Wan, Periodic synchronization control of discontinuous delayed networks by using extended Filippov-framework, Neural Netw., 68 (2015), 96-110. 1, 3.6

[7] J.-D. Cao, J. Wang, Global asymptotic stability of a general class of recurrent neural networks with time-varying delays, IEEE Trans. Circuits Systems I Fund. Theory Appl., 50 (2003), 34-44. 2.4

[8] F. H. Clarke, Optimization and nonsmooth analysis, Canadian Mathematical Society Series of Monographs and Advanced Texts, A Wiley-Interscience Publication, John Wiley \& Sons, Inc., New York, (1983). 2

[9] L. Duan, L.-H. Huang, Z.-W. Cai, Existence and stability of periodic solution for mixed time-varying delayed neural networks with discontinuous activations, Neurocomputing, 123 (2014), 255-265. 1, 3.6

[10] A. F. Filippov, Differential equations with discontinuous righthand sides, Translated from the Russian, Mathematics and its Applications (Soviet Series), Kluwer Academic Publishers Group, Dordrecht, (1988). 2, 2.1, 2.2

[11] M. Forti, M. Grazzini, P. Nistri, L. Pancioni, Generalized Lyapunov approach for convergence of neural networks with discontinuous or non-Lipschitz activations, Phys. D, 214 (2006), 88-99. 2.6

[12] M. Forti, P. Nistri, D. Papini, Global exponential stability and global convergence in finite time of delayed neural networks with infinite gain, IEEE Trans. Neural Netw., 16 (2005), 1449-1463. 2.6

[13] C.-H. Hou, J.-X. Qian, Stability analysis for neural dynamics with time-varying delays, IEEE Trans. Neural Netw., 9 (1998), 221-223. 2

[14] L.-H. Huang, J.-F. Wang, X.-N. Zhou, Existence and global asymptotic stability of periodic solutions for Hopfield neural networks with discontinuous activations, Nonlinear Anal. Real World Appl., 10 (2009), 1651-1661. 2.1, 2.2

[15] B. Kosko, Adaptive bidirectional associative memories, Appl. Opt., 26 (1987), 4947-4960. 1

[16] B. Kosko, Bidirectional associative memories, IEEE Trans. Systems Man Cybernet., 18 (1988), 46-60. 1

[17] S. Lakshmanan, J. H. Park, T. H. Lee, H. Y. Jung, R. Rakkiyappan, Stability criteria for BAM neural networks with leakage delays and probabilistic time-varying delays, Appl. Math. Comput., 219 (2013), 9408-9423. 1

[18] J. P. LaSalle, The stability of dynamical systems, With an appendix: "Limiting equations and stability of nonautonomous ordinary differential equations" by Z. Artstein, Regional Conference Series in Applied Mathematics, Society for Industrial and Applied Mathematics, Philadelphia, Pa., (1976). 2.5

[19] L.-P. Li, L.-H. Huang, Dynamical behaviors of a class of recurrent neural networks with discontinuous neuron activations, Appl. Math. Model., 33 (2009), 4326-4336. 1

[20] H.-F. Li, H.-J. Jiang, C. Hu, Existence and global exponential stability of periodic solution of memristor-based BAM neural networks with time-varying delays, Neural Netw., 75 (2016), 97-109. 1

[21] Y. Li, Z. H. Lin, Periodic solutions of differential inclusions, Nonlinear Anal., 24 (1995), 631-641. 2, 2.3

[22] B.-W. Liu, Global exponential stability for BAM neural networks with time-varying delays in the leakage terms, Nonlinear Anal. Real World Appl., 14 (2013), 559-566. 1

[23] X.-Y. Liu, N. Jiang, J.-D. Cao, S.-M. Wang, Z.-X. Wang, Finite-time stochastic stabilization for BAM neural networks with uncertainties, J. Franklin Inst., 350 (2013), 2109-2123. 1

[24] N. G. Lloyd, Degree theory, Cambridge Tracts in Mathematics, Cambridge University Press, Cambridge-New YorkMelbourne, (1978). 3

[25] R. Rakkiyappan, R. Sasirekha, Asymptotic synchronization of continuous/discrete complex dynamical networks by optimal partitioning method, Complexity, 21 (2015), 193-210. 4.2

[26] D.-S. Wang, L.-H. Huang, Periodicity and global exponential stability of generalized Cohen-Grossberg neural networks with discontinuous activations and mixed delays, Neural Netw., 51 (2014), 80-95. 1

[27] D.-S. Wang, L.-H. Huang, Z.-W. Cai, On the periodic dynamics of a general Cohen-Grossberg BAM neural networks via differential inclusions, Neurocomputing, 118 (2013), 203-214. 1

[28] L.-M. Wang, Y. Shen, G.-D. Zhang, General decay synchronization stability for a class of delayed chaotic neural networks with discontinuous activations, Neurocomputing, 179 (2016), 169-175. 4.2 
[29] H.-Q. Wu, Y.-W. Li, Existence and stability of periodic solution for BAM neural networks with discontinuous neuron activations, Comput. Math. Appl., 56 (2008), 1981-1993. 1

[30] H.-Q. Wu, C.-H. Shan, Stability analysis for periodic solution of BAM neural networks with discontinuous neuron activations and impulses, Appl. Math. Model., 33 (2009), 2564-2574. 1

[31] L.-L. Zhang, L.-H. Huang, Z.-W. Cai, Finite-time stabilization control for discontinuous time-delayed networks: new switching design, Neural Netw., 75 (2016), 84-96. 1 